University of Louisville

ThinkIR: The University of Louisville's Institutional Repository

Electronic Theses and Dissertations

8-2006

\title{
Connecting health concepts and health behavior : the construction of health identity.
}

Valerie French

University of Louisville

Follow this and additional works at: https://ir.library.louisville.edu/etd

\section{Recommended Citation}

French, Valerie, "Connecting health concepts and health behavior : the construction of health identity." (2006). Electronic Theses and Dissertations. Paper 458.

https://doi.org/10.18297/etd/458

This Master's Thesis is brought to you for free and open access by ThinkIR: The University of Louisville's Institutional Repository. It has been accepted for inclusion in Electronic Theses and Dissertations by an authorized administrator of ThinkIR: The University of Louisville's Institutional Repository. This title appears here courtesy of the author, who has retained all other copyrights. For more information, please contact thinkir@louisville.edu. 
CONNECTING HEALTH CONCEPTS AND HEALTH BEHAVIOR:

THE CONSTRUCTION OF HEALTH IDENTITY

\author{
By \\ Valerie French \\ B.A., University of Kentucky, 2004
}

\begin{abstract}
A Thesis
Submitted to the Faculty of the

Graduate School of the University of Louisville

in Partial Fulfillment of the Requirements

for the Degree of
\end{abstract}

Master of Arts

Department of Sociology

University of Louisville

Louisville, Kentucky

August 2006 
CONNECTING HEALTH CONCEPTS AND HEALTH BEHAVIOR:

THE CONSTRUCTION OF HEALTH IDENTITY

By

Valerie French

B.A., University of Kentucky, 2004

A Thesis Approved on

May 25, 2006

By the following Thesis Committee:

Thesis Director 


\section{ACKNOWLEDGEMENTS}

To my family and my roommate who came at me from all angles with endless, unconditional support, patience and love

Hefty doses of time, patience and intelligence from my advisor, Dr. Susan Kelly All of the interviewees, who shared their thoughts and experiences with me Notes and revisions, time and energy from Dr. Patricia Gagne and Dr. Nancy Theriot Kindness and generosity from my dear friends

You four generous individuals - Dr. Kelly, Dr. Gagne, Dr. Theriot, Scott Sallade - who loaned me the books and literature necessary to get this job done

Overwhelming generosity in terms of time, emails, and answers from Jonetta Weber and Lisa Fortwengler to help figure out logistics

Unremitting flexibility and patience from the Alumni staff, which allowed me to actually complete this project. 


\title{
ABSTRACT \\ CONNECTING HEALTH CONCEPTS AND HEALTH BEHAVIOR: THE CONSTRUCTION OF HEALTH IDENTITY
}

\author{
Valerie French
}

May 25, 2006

This thesis is an historical, theoretical and empirical examination of women's health concepts and health behavior in contemporary consumer-oriented American society. It begins with an historical overview of social health movements to illustrate how health concepts are shaped by cultural, social, political, and moral beliefs and practices. It then traces the socio-cultural construction of "health" to understand how this concept is shaped by various social and individual factors.

The qualitative methodology of grounded theory was used to examine empirically how a sample of women defines "health" and what behaviors they engage in related to "health." Non-random convenience sampling was employed to recruit twenty-five women who self-identified as "healthy." Data are derived from semi-structured interviews and verbatim transcriptions.

Findings suggest that dominant cultural images associated with health were important in how the participants talked about health, but when talking about their behavior, "health" was presented as an alternative choice to consumer, aesthetic body ideals that are expressed as oppressive, unhealthy and unattainable. Study participants discussed "health" as having positive moral connotations of being an achievement, which 
was, in part, expressed as contingent upon an appropriate level of self-surveillance and self-responsibility. While study participants expressed recognition of culturally available messages of health, they also exhibited a certain amount of resistance to these messages. Additionally, findings suggest that health concepts and health-related behaviors are negotiated based on habitus. The theoretical concept of habitus is useful in understanding how individuals negotiate health concepts and health behaviors within class-constrained circumstances. The concept of health identity - described as the translation from cultural and social messages of health to individual's health conceptions and health behaviors on a daily, conscious level - may be useful in creating future health promotion, health intervention, and health education programs. 


\section{TABLE OF CONTENTS}

Acknowledgments....................................................................

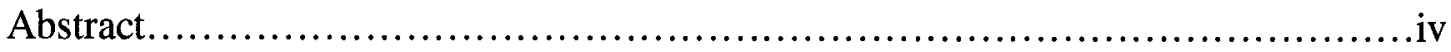

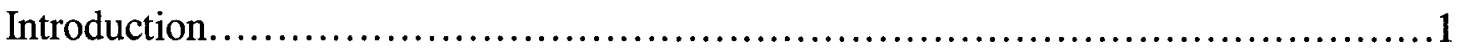

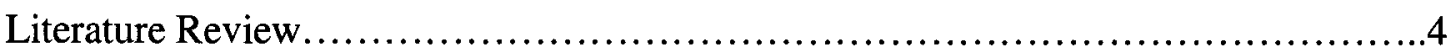

Health-related social movements........................................5

Socio-cultural construction of health concepts............................9

Moral components of health.............................................11

Biomedical model........................................................

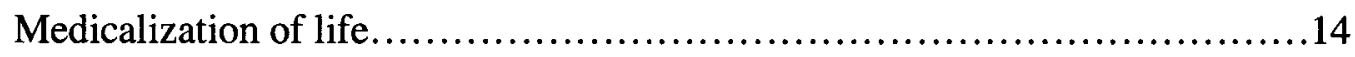

Hospital Medicine to Surveillance Medicine.................................16

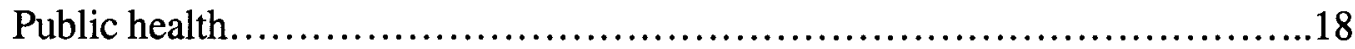

Health promotion........................................................ 19

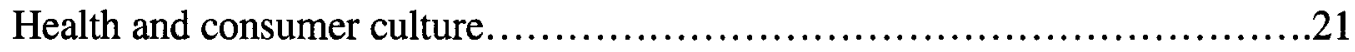

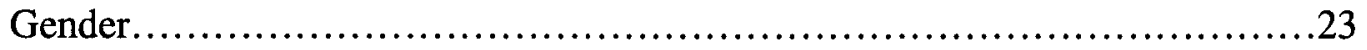

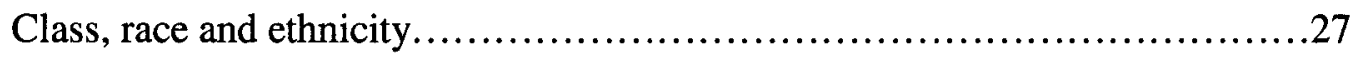

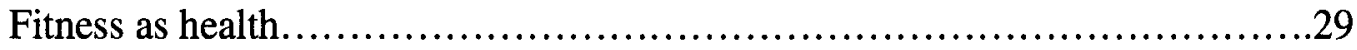

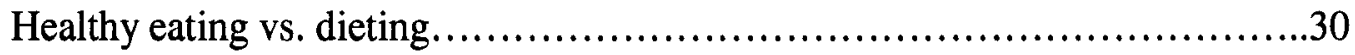




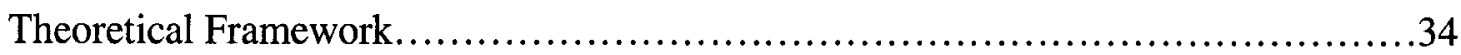

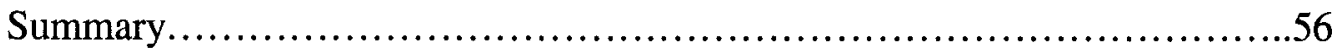

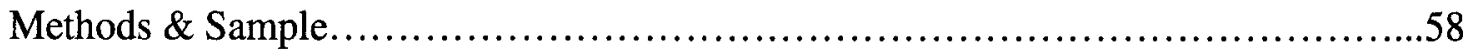

Demographics of sample.............................................62

Findings \& Analysis.........................................................64

Balance and moderation....................................................67

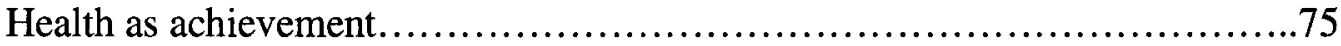

Self-surveillance and self-responsibility .................................79

Consumer culture products and images...................................83

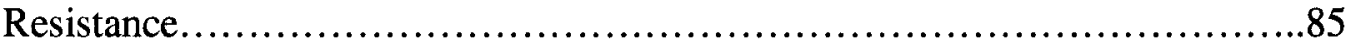

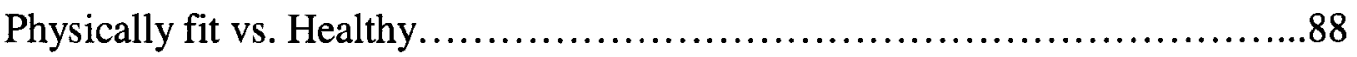

Healthy eating vs. Dieting................................................ 93

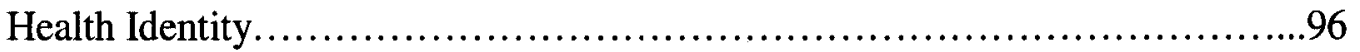

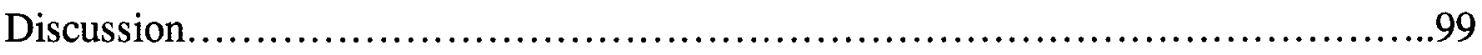

Limits of Generalizability \& Call for Future Research...............................104

Conclusion........................................................................

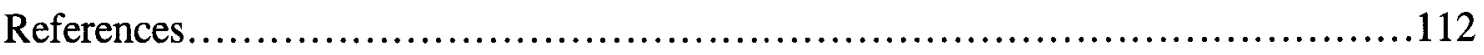

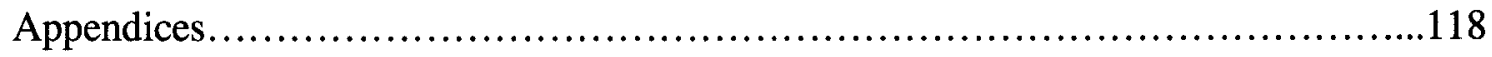

Curriculum Vitae........................................................... 124 


\section{INTRODUCTION}

Concern for health has increased dramatically in the last thirty years (Bunton, 1995). This growing concern comes from individuals, governments, and commercial and media interests (Bunton, 1995). Over the past decade, the growth in health-related industries has been staggering. Total health care expenditures by the United States were $\$ 1.9$ trillion in 2005 and are projected to increase to $\$ 3.6$ trillion in 2014 (Plunkett, 2005). Americans pour billions of dollars annually into the physical fitness industry to purchase fitness-related commodities, such as exercise equipment, gym memberships, yoga videos, and running shoes (Phillips, 2005). In the media, a dramatic increase in health-related magazines, news reports, TV programs, government-funded programs is evidenced (Phillips, 2005), along with websites devoted to issues related to health. Diet-related expenditures, including organic foods, diet books, multivitamins, herbal remedies, and dietary supplements have also skyrocketed. The U.S. organic-food market generated about $\$ 13.8$ billion in sales last year, up from $\$ 11.9$ billion in 2004 and is estimated to reach $\$ 15.5$ billion in 2006 (Gray, 2006). Health promotion campaigns proliferate in contemporary American culture (Lupton, 1995). Health promotion messages target consumers in supermarkets, on the television and radio, in women's and men's magazines (Bunton, 1995), and via Internet websites. It appears that a growing trend in America and worldwide is the quest for health. 
"Health" is a highly salient socio-cultural concept in contemporary American society, yet how people conceptualize the issue of health, the sources of their ideas about it, and the relationship between health concepts and health behaviors are still relatively poorly understood. The World Health Organization (WHO) defines health as complete physical, mental, and social well being. Health, according to the WHO, is not merely the absence of disease or infirmity. And yet, there has been a failure of social scientists to thoroughly investigate the interlocking dimensions of health concepts and health behavior, thereby validating existing tacit assumptions that the terms "health," "healthy" and "healthy lifestyle" conjure up identical concepts in the minds of all individuals and that all individuals engage in similar health behaviors. Little, if any, social scientific research has been conducted that examines individuals' concepts of health and health behavior and how these concepts are shaped by cultural, social, political, and moral beliefs and practices. The purpose of this study is to address this void in the social scientific literature.

This qualitative study draws from the health accounts of twenty-five Midwestern women in an attempt to document women's notions of health, conceptions of health dimensions, influences on conceptualizations of health, and motivations to participate in health-related behavior. While women have been the focus in previous studies that examine cultural body ideals, there has been a scarce amount of research that focuses on women's reactions to these ideals - whether there is acceptance or resistance, and whether these ideals are identified as "healthy" - and in what ways women relate to notions of "health" and "healthy behavior." I used non-random, convenience sampling to obtain my interview sample of informants. My goal in this research is two-fold: to 
contribute to a sociological understanding of health on an individual basis by examining the ways in which health concepts and health behaviors are constructed and enacted, which may thereby provide useful information for designing and implementing health intervention programs, health promotion campaigns, health education, and healthcare providers. With a more informed understanding of how individuals conceptualize health and how these concepts inform health-related behaviors, these programs may reach their audience more effectively, thereby increasing the saliency of their health-related messages. 


\section{LITERATURE REVIEW}

Existing sociological literature on issues related to health is largely focused on an absence of illness (e.g., Litva \& Eyles, 1994), the social construction of illness (e.g., Lorber, 1997), health disparities and inequalities (e.g., Lillie-Blanton et al., 1999; Bolam et al., 2004; Lynam, 2005; Hodgins et al., 2005), healing or recovery after illness (e.g., Frank, 1995), and illness and reconstructing identity (e.g., Charmaz, 1999; Karp, 1999; Sandstrom, 1999; Weitz, 1999). Little literature focuses on issues of health concepts and experiences, what it means to be healthy, or how an individual embodies health understandings or meanings. Indeed, the task of defining health without defaulting to a pathological definition (i.e., as an absence of illness) can pose challenges for researchers. Levin \& Browner (2005) asked a group of anthropologists working from evolutionary, biological and sociocultural perspectives and in diverse geographic regions to examine meanings associated with health and/or to identify social conditions or practices that have contributed to positive physiological and psychological conditions across various cultures or across time. “...In trying to advance this intellectual agenda, we ourselves discovered how difficult it can be to move beyond a pathology-based way of thinking and were struck by the difficulty authors experienced in trying to focus on the subject of health, rather than the absence of disease" (Levin \& Browner, 2005, 746). Although each anthropologist was asked to relate to health in the positive sense, all but one discussed health primarily in terms of the absence of disease. 
Health-related social movements

The history of social health movements illustrates how the concept of health is shaped by cultural, social, political, and moral beliefs and practices. The history of the construction of health concepts has produced various prescriptions for how an individual should care for his or her body, variation in notions of the treatment of bodies, conceptualization of disease and treatment, and the embodiment of health. Notions of health and illness, and the practices for maintaining or attaining health, preventing or curing illness are historically and culturally specific. These notions and practices are reflected in individual embodiment of health.

I will briefly trace the history of health movements in America because they have been significant sources of health concepts. According to Charmaz \& Paterniti $(1999,5)$, "[Health-related] Social movements form to draw attention to, deflect attention from, or change the definitions of health and illness." Attitudes toward the legitimacy and importance of health concepts fall primarily under the force of health movements, as the ideas and beliefs fueling the health movement developed into a set of norms to which the population then adhered (Engs, 2001). Similarly, Freund \& McGuire (1991) emphasize how laypersons' and professionals' ideas about illness are socially constructed; in other words, that these ideas are the result of human activity. The social construction of issues surrounding health is an important component in understanding that a society's medical reality is a social construction (Freund \& McGuire, 1991).

Brown and Zavestoski (2004) define health social movements (HSMs) as "collective challenges to medical policy, public health policy and politics, belief systems, research and practice which include an array of formal and informal organisations, 
supporters, networks of cooperation and media" (Brown \& Zavestoski, 2004, 679). HSMs, as a class of social movements, are described in terms of their influential social impact: HSMs challenge political power, professional authority and personal and collective identity. As these social movements incite individuals' attention, the tenets or goals of the movement consequently affect its participants. The effects of the HSM, then, are wide-reaching, which illustrates how, through the examination of HSMs, researchers may gain insight into this innovative and powerful form of political action that is aimed at transforming the health care system, modifying people's experience of illness and addressing broader social determinants of health and disease of diverse communities (Brown \& Zavestoski, 2004, 691). It is important to note, however, that social movement influence occurs within a larger sociological context in which individuals are influenced by multiple other factors, such as government, capitalist and media sources.

Goldstein (1999) documents the origins of the health-related social movement, which contributes to an understanding of how health-related social movements play a role in the construction of health concepts. Throughout United States history, health-related social movements have influenced concepts of health and illness. The health movement emerged, in part, as a logical step arising from developments in medicine and, in part, as resistance to or avoidance of these medical developments (Goldstein, 1999). Health movements promote societal acceptance of medical and scientific advancements, a legitimacy that is, in itself, a social construction. Health movements have led to various outcomes: from societal support of scientific and technological advancements and a corresponding decline in traditional moral paradigms to a later questioning of science and technology (particularly in regards to its promise for "improvement of life" ever since the 
development and use of the atomic bomb) and resurgence of spiritual and religious paradigms (Goldstein, 1999). Health-related social movements are capable of garnering societal support for various health care concerns, as well as establishing, legitimizing, and popularizing health concepts.

I examine health movements in order to determine how they impact the construction of health concepts. The term "health movement" as it is used here includes both health reform movements (identifiable historical movements) and health-related social movements (such as AIDS awareness campaigns and breast cancer activism). I begin with the historical work of Ruth Clifford Engs (2001), who examines health reform movements. Engs documents three social health reform movements since the founding of the United States, coinciding with the Great Awakenings of the Jacksonian era (18301860), the Progressive era (1890-1920) and the current millennial era (since 1970s). Each of the three movements typically begins with religious revivals, from which significant political, economic, and social changes emerge (Engs, 2001). The specific political, economic and social changes that developed within each period reflect the "tenor of the time" from which they emerged. According to Engs, the Jacksonian era was characterized by a hygienic ideology that was molded by millennialism, romanticism, nationalism, feminism, and educational reforms. The Progressive era was characterized by optimism concerning social improvement based on modern science and the restoration of an America that had been weakened by urbanization, immigration, government corruption, and ruthless capitalism. The current millennial era was shaped by social stresses associated with rapid population growth, significant technological changes, alterations in family structure, and anxieties stemming from the Cold War. 
The first movement explored the "perfectibility of man" through fervent religious practices and, later, therapeutic systems such as hydrotherapy, homeopathy, and botanical medicine. Morality, personal hygiene and diet reform - which grew from the first half of this movement - may influence the construction of health concepts (Turner, 1984). Similarly, sanitation and public health, two concerns more prominent toward the end of the first cycle, influence conceptions of health (Brumberg, 1997; Lupton, 1995). The second movement was significantly influenced by the Christian Science and NewThought religions, and marked by many single-issue crusades. The Eugenics movement, crusades against cigarettes, and the popularity of the "whole man" concept may also affect the ways in which individuals define health (Engs, 2001). The third movement embraced New Age, alternative and complementary health systems (Engs, 2001, 16-17). These trends are influential in health concepts today, as can be seen in the recent popularity of yoga and pilates as models for healthy living (Mees, 2005), the holistic concept of treating the whole person (mind, body and soul) (Gross, 1980), and the increased consumption of organic foods and use of herbal remedies (Gray, 2006).

In another historical examination, Jimenez (1997) discusses the relationship between health concepts and national health care policy. The history of attitudes toward health, Jimenez argues, may illuminate the reasons why the United States is the only industrialized country without a national health care policy system. Health movements in the antebellum period presented health as a private good, achieved through personal struggle and discipline. This conviction guided societal thinking toward health and health concerns during this time. At the beginning of the nineteenth century, however, a vision of health as a collective good emerged within the public health movement. Health of the 
individual was, consequently, viewed as the responsibility of all. Following the Civil War, however, the effect of the public health movement diminished, thereby giving way to reemergence of individual responsibility for health asserted by early health reformers. This ideal set the course for future health care policy in the United States (Jimenez, 1997, 35). The direct link that Jimenez describes illustrates the importance of health movements in guiding and prioritizing health concerns for individuals. Moreover, these health beliefs may then, in the case of national health care policy, have long-term, widespread effects within the health care system.

Socio-cultural construction of health concepts

There are few studies that examine individual accounts of health. Despite its potential for informing how individuals engage in health behaviors, this has been left largely unexplored. However, in one existing project, Radley and Billig (1999) examined American men and women's accounts of health and illness in an attempt to draw out how these accounts differ according to circumstances, intentions and audiences. According to Radley and Billig, health beliefs simultaneously reflect general themes within a culture and specific experiences, feelings, and intentions of the individual. Individuals construct their perceptions of health status as part of their ongoing identity in relation to others and as critical to the conduct of everyday life (Radley \& Billig, 1999, 19). The findings present a double existence of ideas related to health and illness: on one hand, they are the means by which individuals maintain and define themselves as useful for society, while on the other hand, these accounts portray a world of experiences that individuals claim for themselves alone. Important to my own research is this notion of health as part of an 
ongoing negotiated identity. However, the researchers did not examine how concepts of health are variously gendered, and how gender impacts the experience of health, the construction of health concepts and the enactment of health behaviors.

Manderbacka (1998) examines the ways in which individuals respond to the selfrated health question that appears on many medical questionnaires, which asks the person to assess his or her health on a scale from "excellent" to "very poor." She asks this question in an interview-based setting to elicit conversation about health concepts and the individual's criteria in self-rating health. Through semi-structured interviews with 42 participants, Manderbacka explored the health that participants defined as "excellent" or "good" or "poor." She wanted to determine how these assessments were made. Several determinants of self-rated health emerged from her data, such as the respondent's medical health status and its consequences on the individual's daily functioning; physical fitness; illness and disease symptoms; and psychological well-being. Health was discussed primarily as an absence of illness, yet participants also connected health to their personal experience and life situation, and as a result of personal action (Manderbacka, 1998, 145). The results of this study suggest that health is assessed holistically and as situated within life experiences. In addition to the medical model of health, Manderbacka argues, health assessments should adopt messages of health promotion and healthy "lifestyles." This study informs my own in its examination of health concepts and in its focus on eliciting these health concepts from individuals. I would like to add the component of gender to examine how gender plays a significant role in constructing an individual's health concepts and health behaviors. 
In another examination of the construction of health and illness concepts, Hodgins et al. (2005) explore the perceptions of illness causation and health inequalities of Traveller women. Travellers are a small indigenous minority in Ireland whose nomadic tradition sets them apart from "settled" people on several levels, including social and health disadvantages. Forty-one participants responded to a vignette in a focus group setting. Hodgins et al. conclude that there is noticeable complexity in lay perceptions of ill-health and health inequalities and that their understandings of health and the factors that determine it are deeply embedded in the social context of their lives and their ethnic identity. The finding that the participants' health concerns are specific to their social context and ethnic identity is supports the development of culturally-sensitive health services. This study of Traveller women's perceptions of ill-health contributes to an overall understanding the dimensionalized and socially-situated components of health concepts. The emphasis on social, ethnic, racial, gender and cultural factors in the construction of health concepts is vital to my own exploration of women's health concepts and health behaviors in North America.

Moral components of health

In pre-modern society, health and morality were fundamentally united in practice and in theory (Turner, 1984). Pre-scientific understanding of behavior emphasized either the causal role of divine forces or the absence of external regulation and the power of the individual's free will (Goldstein, 1999). Deviance was seen as the result of moral weakness or sin, to which the individual was to provide an "appropriate response" such as prayer, pastoral guidance, moral effort, or in their absence, guilt (Goldstein, 1999, 34). 
Understandings of why an individual was either ill or healthy were taken from the individual's moral standing.

In the early nineteenth century, health reformers relied heavily on religious ideologies to inform the public about health issues and concerns, as well as to motivate the behavior of these individuals (Engs, 2001). These health reformers, known as "Christian physiologists," believed they were a part of the process by which "God was restoring the purity of the ancient order of things and would bring the nation back to a gilded age of better health. Their aim was to make America a godly, sober, and healthy place" (Engs, 2001, 54). Total abstinence from alcohol and other stimulants, and, particularly, the reformers' reliance upon moral and biblical beliefs as the basis for this argument (Engs, 2001) meant that health became a moral crusade. Disease and illness were connected specifically to the individual moral standing (Turner, 1984).

The emphasis on an individual's activities and behaviors as cause for moral corruption and disease meant that scientific, laboratory-based advancements in medicine were slow to take hold. Rather than seeking out scientific solutions, individuals during this pre-modern time legitimized the connection between morality and health. Thus, although scientific discoveries revolutionized thinking as early as the seventeenth century by introducing ideas of matter, new applications of mathematics to physics, and new experimentation methods (Weiss \& Lonnquist, 1997), these revolutionary ideas were slow to be incorporated into understandings of health. Much skepticism still existed surrounding scientific and laboratory-based medicine despite these scientific advancements, which led to a continued reliance on home remedies and "domestic medicine" (Weiss \& Lonnquist, 1997). Many physicians continued to favor the humoral 
theory of disease, which proposes health as a balance of the four elements in the body (blood, phlegm, yellow bile, and black bile) and a balance of the individual with the environment (Weiss \& Lonnquist, 1997).

Biomedical model

In the late nineteenth century, Enlightenment science began to replace alternative methods and systems for understanding and dealing with health and illness (Goldstein, 1999). The power of the medical model grew, fostered by a "perceived incompatibility" between religious and scientific views (Goldstein, 1999). The biomedical model is frequently cited as the dominant approach to disease in Western medicine (Gabe et al., 2004). Four primary assumptions shape the biomedical model: the presence of disease, its diagnosis, and its treatment are all completely objective; only medical professionals are capable of defining health and illness; health and illness should be defined on the basis of physiological malfunction; and, health is defined as the absence of disease (Weiss \& Lonnquist, 1997, 106-7).

With significant discoveries in the 1880 s that causally linked specific microorganisms to specific diseases (e.g., the tubercle bacillus to tuberculosis and the vibrio cholera to cholera), this new biomedical model began to gain scientific legitimacy and popularity (Gabe et al., 2004). Additional advancements within the medical field required expertise and specialized training. From roughly 1920 through the 1940 s, infectious diseases were ruthlessly attacked, an initiative that revealed one "magical bullet" after another and, consequently, produced peak pharmacological success (Weiss \& Lonnquist, 
1997, 28). Patients began to expect magical results with each new discovery of cures for bacterial infections, lifesaving surgeries, and technological advancements (Engs, 2001).

Biomedicine aims to connect specific diagnoses with specific causes, which has implications for current conceptualizations of health. Consistent with the biomedical model, current conceptions of doctor visits resemble those that emerged in late nineteenth century: in one-on-one conferences, the doctor searches for an accurate diagnosis and effective treatment. Physician attention is focused on diagnosing the individual body. A diagnosis, in terms of manifestations and consequences, personalizes the illness and connects it directly to the individual body, thereby separating it from broader implications (Mechanic, 1999). Individual manifestation of disease has been cited as an influence on the individualization of modern medicine: "Doctors were increasingly oriented towards individual 'presentation,' rather than tackling the complexities and heterogeneity of the patient's familial, social or moral worlds. The medical model can be seen, therefore, as reinforcing individualism as a dimension of modern experience" (Gabe et al., 2004, 126). Current conceptualizations of health are influenced by the biomedical model, specifically in terms of diagnosis and treatment of individual bodies, and bodies cured via medical treatments separate from familial, social or moral concerns.

\section{Medicalization of life}

The medicalization of a wide range of social phenomena characterized what is often termed a "medicalization of life." Medicalization may be defined as "the process of legitimating medical control over an area of life, typically by asserting and establishing the primacy of a medical interpretation of that area" (Freund \& McGuire, 1991, 213). 
Medicalization of life meant that various life events or conditions came to be defined as medical problems, such as childbirth and alcoholism, which were previously considered part of nature, or issues related to morality, sin, deviant behavior or character (Conrad \& Schneider, 1992). As science and technology developed, so too did the desire for rational, objective, thinking about health. A new hospital-based form of medicine emerged in the late eighteenth century that was based on the four innovations of structural nosology, localized pathology, physical examination and statistical analysis (Williams, 2003, 11).

The ascendancy of medical authority is an important social development. The history of the rise of professional scientific medicine is significant for how current cultural understandings of and societal approaches to health developed. The increased credibility and legitimacy was promoted by, for example, the American Medical Association in 1847, which formed in an effort to promote the professionalization of medicine (Weiss \& Lonnquist, 1997). Paul Starr (1982) examines the medical profession in America, tracing it from its poorly regarded beginnings to its ascendancy as a powerful, prestigious occupation. These medical professions have developed social and cultural authority to maintain their prestigious positions. Social authority refers to authority that has been built into laws or rules; while cultural authority refers to "the probability that particular definitions of reality and judgments of meaning and value will prevail as valid and true" (Starr, 1982, 13). Significantly, while social authority may be legislated, cultural authority is awarded when professions successfully persuade the public that they are worthy of this authority. Thus, by the 1920 s, medical authority was clear: "its prominent position and ability to control the health care system were firmly established" (Weiss \& Lonnquist, 1997, 31). 
Hospital medicine to surveillance medicine

Armstrong (1995) documents a significant transition within medicine: from Library Medicine to Bedside Medicine to Hospital Medicine and, finally, to a new Surveillance Medicine. Library Medicine described a period that prioritized the classical learning of the physician over knowledge of specific illnesses; Bedside Medicine emerged as physicians began treating patients' illnesses, concentrating specifically on documenting symptoms (Armstrong, 1995, 393). Hospital Medicine emerged with the dawning of hospitals in Paris at the end of the eighteenth century. Armstrong $(1995,393)$ describes Hospital Medicine - or pathological medicine, Western medicine, or biomedicine - as the dominant model of medicine in the modern world. Contemporary society has produced a new form of medicine: Surveillance Medicine.

The transition from Hospital Medicine to Surveillance Medicine is "a transition symbolized, in the current era of health promotion, by the strategic shift to a spatiotemporal calculus of risk factors, crystallized in the moral pursuit of health through lifestyles" (Williams, 2003, 17-18). Surveillance Medicine involves health promotion techniques, thereby recognizing "that health no longer exists in a strict binary relationship to illness, rather health and illness belong to an ordinal scale in which the healthy can become healthier, and health can co-exist with illness; there is now nothing incongruous in having cancer yet believing oneself to be essentially healthy...But such a trajectory towards the healthy state can only be achieved if the whole population comes within the purview of surveillance: a world in which everything is normal and at the same time precariously abnormal, and in which a future that can be transformed remains a constant possibility" (Armstrong, 1995, 400). "Trajectory towards the healthy state" requires 
individuals to assume personal responsibility for their health. This responsibility equates to conscious, daily efforts, self-regulation, and self-control.

In contemporary understandings, health has become something fragile and inherently contingent. Inherent contingency is "embraced by the novel and pivotal medical concept of risk. It is no longer the symptom or sign pointing tantalisingly at the hidden pathological truth of disease, but the risk factor opening up a space of future illness potential" (Armstrong, 1995, 400). The extracorporeal space - or, the activities and environment of the individual - becomes increasingly significant. This space, often represented within the context of a person's "lifestyle," is targeted by the "medical eye" as a way to predict future illness: "Symptoms, signs, illnesses, and health behaviours simply become indicators for yet other symptoms, signs, illnesses and health behaviours...The problem is less illness per se but the semi-pathological pre-illness atrisk state" (Armstrong, 1995, 401). An individual who smokes, for instance, learns that this action puts her at an increased state of risk. Smoking is an indication that this individual is at an increased state of risk for future problems and illnesses, such as "smoker's cough," lung cancer, bad breath, and mouth cancers. Individuals teeter between health and illness and learn that specific practices may influence their state of health.

Arthur Frank (1995) conceptualized what he calls a "remission society," a community built upon the idea that health is a contingent characteristic; that is, the insidious notion that where there is health, there is illness. Frank describes this society as made up of "all those people who...[are] effectively well but could never be considered cured. These people are all around, though often invisible...Members of the remission 
society include those who have had almost any cancer, those living in cardiac recovery programs, diabetics, those whose allergies and environmental sensitivities require dietary and other self-monitoring....and for all those people, the families that share the worries and daily triumph of staying well" (Frank, 1995, 8). Though medicine offers pharmaceuticals and treatments, the level of health is a personal issue; health is a daily accomplishment, a project toward which a person must consciously work, thereby devoting time, energy, and money on a consistent, daily basis. Without these efforts, an individual may lose his or her health. To "be healthy" is not a given - it is contingent. Its achievement requires constant attention.

Public health

The history of public health is often divided into the "old" public health and the "new" public health (Gabe et al., 2004). The "old" public health, which occurred prior to the Enlightenment, is constituted primarily by two kinds of public health measures: emergency measures in response to epidemic outbreaks and everyday municipal details, such as waste disposal (Lupton, 1995). Public health during the Enlightenment period, lasting roughly from the end of the seventeenth century until late eighteenth century, was a transitional period; during this time, rational, scientific thought began to gain legitimacy, thereby having a similar effect on health practices - public health movements adopted rational, progressive, cause-and-effect practices (Lupton, 1995). The "new" public health is a term popularized in the 1980s, which may be defined as "the resurgence of interest in the social, economic and environmental determinants of health and the 
advocacy of healthy public policy, rather than health services, to improve the health of populations" (Gabe et al., 2004, 233).

New public health has shaped the motivations for participating in health-related behaviors. Referring to the "new" public health, Lupton $(1995,4)$ states: "In this secular age, focusing upon one's diet and other lifestyle choices has become an alternative to prayer and righteous living in providing a means of making sense of life and death. 'Healthiness' has replaced 'Godliness' as a yardstick of accomplishment and proper living.” Public health movements attempt to pull from a variety of human sciences psychology, demography, sociology, sexology, economics - in order to categorize and construct the human actor within a comprehensive health-conscious environment (Lupton, 1995).

Health promotion

Health promotion may be defined as planned activities (e.g., surveys, screening and public health campaigns) that are designed to "monitor, regulate and induce good health practices in both individuals and the population in general" (Gabe et al., 2004, 155). Health promotion logic focuses on individuals in an effort to persuade and motivate each individual to "uncover their true state of health, to reveal their moral standing and indeed shape their true selves by strategies of bodily management" (Lupton, 1995, 138). Oftentimes, adherence to governmental discourses of health promotion is articulated in people's discourses at the conscious level (Lupton, 1995) such that health becomes a conscious, immediate set of tasks. 
Health promotion encourages self-responsibility and a personal commitment to consistent investment in the project of achieving, improving or maintaining health (Engs, 2001; Street, 2004). While health has historically been defined largely based on issues of mortality whereby an individual "had health" unless stricken with a severe illness or on his or her deathbed (Morantz, 1984), health promotion logic in contemporary society has attempted to teach individuals that health is experienced as an achievement; in other words, health is pursued as a project (Doel \& Segrott, 2003; Gross, 1980; Shillingford \& Shillingford Mackin, 1991). This logic teaches individuals that health is achieved through discipline; individuals learn that they must invest time, money, energy, and resources into the project of health.

In her research on the construction of smoking in advertising, Annette Street (2004) refers to the issue of individual responsibility and self-governance in health promotion strategies, stating that population regulation is the reason for individual compliance to health behaviors. The health of each individual is placed directly in his or her hands. "Compliance is not only structured by regulation but also by a new industry in health promotion...Despite its multifactorial beginnings centred in social concepts of collaboration, engagement with citizens and accountability, much modern health promotion has become a strategy to focus on individual responsibility based on the belief that individuals can control their own health and health outcomes" (Street, 2004, 234).

Health promotion programs have affected the ways in which health is conceptualized. Promotional messages have encouraged elements of individual responsibility, self-governance, and control as part of individual health concepts, and as a result, a person's notions of what health is and how health is embodied may reflect these 
elements. In an examination of worksite health promotion, Conrad (1999) explores the upswing in worksite health promotion that has occurred in the past decade within the United States as part of the growing national interest in both disease prevention and health promotion. By combining educational, organizational and environmental activities, worksite health promotion aims to "support behavior conducive to the health of employees and their families" (Conrad, 1999, 263). These activities consist of health education, health screenings and interventions designed with the goal of behavioral change. Worksite health promotion, which began to emerge in the 1970s, has developed primarily without physician input, but has cited substantial benefits, including decreases in employee absenteeism, turnover and worker compensation (Conrad, 1999). Findings suggest, however, that worksite health promotion programs, largely an American phenomenon, may be less successful than is claimed; thus, while the potential for concrete, documented success is there, it remains as a hope for the future (Conrad, 1999).

Health and consumer culture

In contemporary consumer-oriented American culture, health and aesthetics are connected in a particular, consumer nexus. Bodily care and aesthetics are often negotiated within the marketplace. The strategies for this type of aesthetic health care are situated within consumer culture: "Self preservation depends upon the preservation of the body within a culture in which the body is the passport to all that is good in life. Health, youth, beauty, sex, fitness are the positive attributes which body care can achieve and preserve" (Featherstone, 1982, 26). Individuals may learn through cultural messages that in order to 
look good and present a healthy, youthful body to others, consumption of health-related commodities is necessary.

In a critical analysis of the relationship between health and consumer culture, Bunton (1995) examines the role of commodities in a newly emerging "healthism," which has become a significant part of Western contemporary consumer culture. Healthism promotes images of youthfulness, vitality and energy, and in an effort to achieve these ideals, citizens of Western capitalist societies rely on health-related commodities, the availability of which is seemingly endless (Bunton, 1995). The primary criticism is the capitalist undertones disguised as an effort to increase health, as Bunton claims the new health promotion campaigns are "increasingly indistinguishable" from other forms of product advertising and are often developed in collaboration with product promoters and health product advertising (Bunton, 1995).

Doel and Segrott (2003) examined complementary and alternative medicine in British mass media, in which they found messages that emphasized health as holistic. The researchers found that nearly every aspect of one's daily practice is identified as part of health management, from personal relationships to financial strategies to time management - all of which have the potential to either help or hinder health achievement (Doel \& Segrott, 2003, 135). Individuals learn routes to successful health, oftentimes relying on external instruction and direction, products and materials, and information and knowledge to achieve a healthy lifestyle. In this examination, readers were called upon to shape every aspect of their lives into more healthy prototypes, an ideal that is connected to the belief that health is holistic (Doel \& Segrott, 2003). Such messages, in turn, have important effects on the specific goals and initiatives an individual adopts in his or her 
pursuit of health, as well as the ways in which he or she conceptualizes health. Rather than learning about health primarily through familial traditions, Doel and Segrott illustrate how individuals are largely socialized by media sources. The researchers examine health as a concept negotiated within consumer culture whereby media sources convey marketed efforts to sell products rather than efforts to convey what is "really" good for individuals.

Gender

Gender is an important consideration in any discussion of health. Because many health promotional messages target women as the facilitators and regulators of healthy living - which responsibility is enacted through their roles as wives and mothers women are consequently assigned a crucial role in the health of contemporary America. Moreover, the connection between aesthetics and bodily health care illustrates an important gendered component in the construction of health concepts. How an individual incorporates this connection between aesthetics and bodily health care is gender-specific, which further emphasizes the need for the consideration of gender.

Lorber (1997) examines the construction of the body through the gendered social practices that produce social bodies through life and death (Lorber, 1997, 3). By placing the social constructions of gender and illness side by side, Lorber is able to gauge how these constructions simultaneously work on bodies: transforming them from physical bodies into social ones. Lorber's $(1997,7-8)$ gender analysis illustrates how gender is built into "almost every aspect of illness in modern society: risks of and protections from different diseases, the perception and response of the patient to symptoms, the 
organization and delivery of health care, the politics of diagnosis, funding priorities, the questions asked by clinical and scientific researchers, and the knowledge and meaning of diseases and their treatment." Lorber finds that gender affects illness experiences such as doctor visits, complaints of symptoms to physicians, old age and dying. All through the human experiences of birth, death, disability, and illness, gender (as well as class, ethnicity, cultural phenomena, social status) plays a role in one's experiences (Lorber, 1997).

Health promotion messages target men and women differently (Lupton, 1995). Since the nineteenth century, public health strategies have routinely positioned women as nurturing, caring mothers who are the guardians of their family's health and, by extension, the guardians of the health of the nation (Lupton, 1995). Health promotional messages target women as the dietary regulators of their partners and offspring according to the dictates of health guidelines, the monitors of their partner's weight and exercise habits, and the supervisors of their children's cleanliness and vaccination history (Lupton, 1995). And finally, health promotion relies on emotional appeals to incite women's anxiety about their outer appearance; through direct appeals, women learn to monitor the attractiveness and youthfulness of their bodies (Lupton, 1995). In America, health is constructed as a specific "experience" for women: women are responsible for taking care of others and they are responsible for shaping their outer appearance to represent attractiveness and youthfulness. The messages that women receive from health promotion appeals has an important gendered effect on the ways in which health is conceptualized and enacted through health behaviors. 
The centrality of the body has become an increasingly pervasive notion in contemporary society. Joan Jacobs Brumberg (1997) explores the ways in which women are encouraged to obtain a beautiful, perfect body - a project, she states, that shapes the concerns of many women. In an historical analysis, Brumberg captures the patterns in girls' ways of thinking about their bodies. In the $19^{\text {th }}$ century, girls were focused somewhat on bodily shape and composure (corsets), yet their primary focus was on inner strength, goodness of heart and spirit, and purity. Over time, girls have become increasingly concerned with exercising control over their bodies in an attempt to shape them into an image that reflects cultural ideals. Brumberg identifies the slow, steady, and powerful takeover of the visual element of our culture, consequently demanding more and more of our attention. Today, girls equate bodily shape and appearance with identity. Girls are paying an ever-increasing amount of attention to their own individualized body projects, using the consumer culture as their guide. In turn, the consumer culture continues providing girls with "ideals" to which they become obsessed with satisfying from sanitary menstrual cycles to unblemished skin to perfect breasts. With an evergrowing list of requirements, American girls have learned that the perfection of their bodies is their first and foremost project. Under these cultural pressures, girls have consequently become increasingly anxious and unsure of their bodies and, in turn, of themselves. Brumberg (1997, xxi) states that beauty is contingent upon the investments a woman makes into this project: "At the end of the twentieth century, the body is regarded as something to be managed and maintained, usually through expenditures on clothes and personal grooming items, with special attention to exterior surface - skin, hair, and contours." This ever-growing concern with obtaining a perfect body may have important 
implications for the ways in which women conceptualize their bodies as a mutable entity which they may shape into cultural ideals. In contemporary American society, the fit, lean and toned body is recognized as an emblem of "health." In response to this cultural ideal, women in contemporary American society may be persuaded to shape their bodies into this cultural ideal in an effort to present themselves as "healthy."

Similarly, Bordo (1993) looks at the slender body as a "healthy body" in her analysis of feminism, Western culture and the body. Slenderness becomes an important component of a discussion on health issues, as contemporary consumer-oriented American society has equated "slenderness" with "health," particularly for females (Bordo, 1993; Lowe, 1999). Bordo (1993) states that the slender body is a gendered body (as the slender body is typically displayed as female) and that this gendered body is never neutral; thus, "slenderness [is] a contemporary ideal of specifically female attractiveness" (Bordo, 1993, 205). Promotional and marketing messages of slenderness are disproportionately disseminated to women than men, thereby producing significant results: "Women are much more prone than men to perceive themselves as fat. And, as is by now well known, girls and women are more likely to engage in crash dieting, laxative abuse, and compulsive exercising and are far more vulnerable to eating disorders than males" (Bordo, 1993, 204).

The obsession with managing diet and hunger is examined in Lowe's (1999) account of calorie counting and dieting among college-aged women in the 1920s. She found that prior to World War I, girls at Smith College were desirous of weight gain; food played an important role in their lives. As students, their families and college officials perceived weight gain as a sign of health (Lowe, 1999, 173). By the 1920s, 
however, girls were encouraged to do away with "robust eating" in favor of calorie counting and conservative eating in order to obtain, or maintain, a slimmer figure. Lowe's historical account recognizes the body as a social construct; she focuses on the moment in history when women's bodies were specially targeted. Her study describes the relationship between cultural discourses about the female body and women's attitudes toward their bodies. She found an historical moment when dieting became integral to college women's conceptions of their bodies, particularly when it came to be recognized as a tool for changing them (Lowe, 1999, 173). For the women at Smith College, dieting emerged as a tool to shape their appearance in the post-World War I era. Men and women experience contemporary American ideals of thinness differently: for women, these pressures are much greater (Freund \& McGuire, 1991; Bordo, 1993). How women respond to these cultural pressures - whether they resist or subscribe to these pressures is of particular interest. Further, how do women who self-identify as healthy interpret these cultural ideals of slenderness - for example, as healthy, unhealthy, attainable or unattainable? Is the quest for slenderness viewed as a healthy practice?

Class, race and ethnicity

Just as gender is a powerful influence on life experiences in the United States, so too are the social factors of class, race and ethnicity (Meinert, 2004; Lillie-Blanton et al., 1999; Freund \& McGuire, 1991). An individual's class and corresponding access to resources plays a role in his or her knowledge of particular health concerns and access to health-related resources (Meinert, 2004). The way in which an individual may come to define and engage in health-related behaviors is a "result of the complexity of experience 
in the world, rather than as a result of formal rules or individual will...health practices...should be understood as interplay between locally defined resources and competences to mobilize them" (Meinert, 2004,13).

Access to resources also shapes a person's lifestyle. If she is raised in an environment where financial resources are scarce, she may not value the investment of extra money to purchase organic foods, for example. "Individuals, socialized within a particular lifestyle, develop a preference or a taste for that lifestyle, leading to a reproduction of that lifestyle" (Singh-Manoux \& Marmot, 2130). These preferences become naturalized for that individual, though they may be more accurately defined as structured by sociological dimensions such as class and power.

Motivational messages to eat healthily and participate in exercise, as well as the opportunities to do so, are socially distributed (Freund \& McGuire, 1991). This social distribution has very real effects on how women are targeted by health messages, as well as how they are able to engage in these activities: "Obesity is not as heavily stigmatized in the lower class. Dieting and fitness rituals are primarily a middle- and upper-class phenomenon, not motivated simply by a concern for better health" (Freund \& McGuire, 1991, 47-8). While some cultures value fat as a sign of prosperity, in middle-class American culture, fat symbolizes a lack of self-control (Freund \& McGuire, 1991). The consideration of both class and race in an individual's construction of health concepts and health behavior is vital, as these social factors play a significant role in this socially situated process.

The intersection of race, class and gender may create increased barriers to health. These intersectional influences have substantial effects on a person's access to health 
resources, understandings of health, and participation in health behaviors. Women of varying racial, ethnic, educational, religious, physical ability, and age backgrounds will encounter society as a matrix of variables that either dominate them or don't (Collins, 2000). A middle-aged White, middle-class woman with a college degree will encounter society differently than an elderly Asian American, upper-class woman without a high school diploma. These variations will then affect how these women conceptualize health and participate in health-related behaviors. Knowledge of health reflects positioning within matrices of power and domination. How women of varying background live health will vary with the matrix of societal variables that they encounter.

Fitness as health

The fit, slender body as aesthetically pleasing, and therefore desirable, was popularized early in the twentieth century (Lowe, 1999). In addition to dieting as a tool for slimming the body, fitness became an additional route to obtaining the dominant cultural ideal of a slim body (Engs, 2001). The crusade for fitness, which included exercise, health concerns and diet, was popularized in the "fitness and wellness movement," which began around 1965 (Engs, 2001). The federal government began promoting fit bodies as healthy, desirable bodies when many Americans were discovered "unfit" for military service in World War II (Engs, 2001). President Dwight D. Eisenhower created the Presidential Council on Physical Fitness in 1955 and, two years later, the President's Council on Youth Fitness. In the next administration, President John F. Kennedy targeted overweight children in his partnership with the Council on Physical Fitness (Engs, 2001). From 1970 until 1990, the quest for fitness surged into a massive 
movement, which consequently spurred many "crazes" and trends: running was popularized with the 1966 publication of Runners World, an aerobics craze began in 1968, a "wellness" approach that promoted disease prevention and health maintenance began in the early 1980s, and a push for "personal responsibility for health" was laced within preventive and health promotion messages (Engs, 20001, 249-251).

In health promotional messages, "fit" bodies are equated with healthy, regulated ones (Lupton, 1995). Engaging in regular exercise, particularly if this exercise is devoted solely to body maintenance (rather than, for instance, enjoyment purposes), indicates that this individual has a high capacity for self-regulation and self-control (Lupton, 1995). Health promotion messages that encourage this type of regulation and control of the body for the purposes of "fitness" are influential, yet are not necessarily stable: "The ways in which discourses are taken up and integrated into self-identity are at least partially contingent on the flux of individuals' positions in the workforce, in the lifecycle and the interaction of institutions such as the economy, the family, the school" (Lupton, 1995, 149). Thus, individuals in contemporary American society are surrounded with messages of "fitness as health," yet the ways in which this fitness is experienced on an individual level will be determined in part by that individual's social environment.

Healthy eating vs. dieting

The act of eating and the foods that are consumed have, over time, had different societal meanings and purposes (Shilling, 2005; Turner, 1984). While eating is an activity which has a basic physiological purpose, this act is simultaneously heavily mediated by culture (Turner, 1984, 176). A wide variety of food can be consumed by people while 
still providing adequate nutrition. This variety, Shilling $(2005,154)$ argues, means that social institutions can dictate this consumption without harming the nutritional needs of citizens. The enormous variety of foodstuffs consumed by people means that food selection has as much to do with social taboos and cultural preferences as it does with the need to satisfy hunger and provide nutrition for the body (Shilling, 2005, 154). Due to this malleability, the culturally available messages of "healthy eating" have gone through numerous changes.

Traditional societies were characterized by eating communities, which instilled collective, communal and often moral concepts of shared eating (Shilling, 2005). Holy Communion, for instance, involved the "digestion" of the individual into the sacred community. Modern societies are characterized by individualization. Consequently, Shilling $(2005,155)$ proposes that modern societies encourage individual relationships between eating and bodily identity. The role of food in this "project of the self" is evident in the marginalization of the meal and increasing consumption of fast food, treats, and other foods symbolically associated with enjoyment and contentment. Cultural transformations in the "meaning" attached to eating have affected individuals' relationship with food. Shilling $(2005,162)$ argues that the relationship women have with food in contemporary society is highly damaging to their self-identities and their "ability to engage in the generation of intrinsically satisfying relations of sociality." This particular relationship women have with food is an outgrowth of modern society, Shilling argues. The variability in cultural meanings illustrates the impact of society on the individual's food choices and nutritional intake, as well as the concept of "healthy eating" and how this concept may vary depending upon gender. 
In her historical examination of health reform movements, Engs (2001) found several instances of dietary teachings. In 1837, William Alcott and Sylvester Graham formed the American Physiology Association (APA), which proposed specific dietary recommendations, thereby influencing the nutritional intake of its followers. For example, Graham and Alcott believed that anything that could potentially harm the body should be avoided. Thus, "healthy eating" as conceptualized by Graham and Alcott - and adhered to by their followers - was abstinence from most meats, salt and salt pork, gravies, fried foods, butter, pastries and all stimulants (tea, coffee, wine, beer, and spices) with meals eaten six hours apart (Engs, 2001, 59). By 1910, however, this strict vegetarianism began to wane. Sports and athletics were conceptualized as related to diet and, because a high-meat diet was believed to be important for physical progress, dietary habits changed according to this emerging belief (Engs, 2001, 114). In the last decades of the twentieth century, a new dietary regimen emerged. In 1960, eating patterns changed in middle-class America: while previously, meals were typically eaten at home and consisted primarily of meat and potatoes, with some vegetables and dairy products, by the 1970s, fast-food restaurants, frozen entrées, and precooked items boomed. With increased convenient foods came increased levels of fat, sugar and foods with little nutritional value, a phenomenon that spurred campaigns against these unhealthy foods. As a result, numerous concepts of "diets" have been proposed by societal institutions, such as low-fat diets, low-carbohydrate diets, low-sugar diets, and the raw foods diet (all of which propose different recommendations concerning fat, sugar, meat, dairy, and alcohol consumption). Cultural changes in eating habits and attitudes toward certain 
foods consequently influence personal concepts of eating, particularly in terms of what foods are considered healthy versus unhealthy.

Concepts of health and health behavior are shaped by cultural, social, political, and moral beliefs and practices. Social health movements demonstrate how these concepts change over time and place. Traditional concepts of health led communities to connect divine forces to the state of a person's health. Moral components of health still inform current, contemporary concepts of health. Severe illness, illness recovery, and purity of body are experienced within a moral or spiritual context for some individuals. As science and technology emerged, however, society turned toward scientific cause-andeffect explanations for disease and illness. Reliance upon doctors and medical professionals is clearly present in contemporary notions of health and health care. Public health and health campaigns began steering societal concepts of and participation in health and health behavior. Self-responsibility, self-surveillance, and self-control are concepts that underlie these campaigns, consequently influencing the ways in which health is conceptualized. Health has come to be identified as a contingent entity which may be achieved if an individual constantly works toward and takes responsibility for this goal. Gender, class, race, and ethnicity are all social factors that influence health concepts and health behaviors in terms of health embodiment, responsibility, access to resources, priority of health, and so on. Individual health concepts are influenced by cultural messages of "healthy eating," and "fitness." 


\section{THEORETICAL FRAMEWORK}

My investigation of health concepts and health behaviors is informed by the theoretical work of Michel Foucault, Pierre Bourdieu, Theodor Adorno, Susan Bordo, and Patricia Hill Collins. The social, cultural, political, and moral influences on the construction of health requires an investigation that explores the relationship between populations and individuals, power dynamics, social organization and societal factors such as gender, race and class. The following framework seeks to assess these societal complexities in order to gain a better understanding of the ways in which socially situated health concepts and health behaviors are constructed over time and location, negotiated within contemporary consumer-oriented American culture, and are gender, race and class specific.

The theoretical work of social theorist Michel Foucault (1926-1984) is useful for examining health concepts. Of particular interest here is the way in which his theoretical concepts intertwine with the investigation of health; specifically, the dimensions that form individuals' conceptions of health and the health-related behaviors enacted in their daily lives. Foucault's theoretical work aids in an understanding of the ways in which "health" is conceptualized and practiced by individuals as well as society at large. In my examination, I am especially interested in Foucault's concentration on the ways in which experience is ordered and realized, the relationship between truth, knowledge and power, and his concepts of bio-power and subjectivity. 
Foucault applied historical analysis as an interpretive sociological methodology. Such an interpretive understanding requires the researcher to "undertake the hard historical work of diagnosing and analyzing the history and organization of current cultural practices... resulting [in an] interpretation [that] is a pragmatically guided reading of the coherence of the practices of the society" (Dreyfus \& Rabinow, 1983, 124). Foucault examines how the experiences of individuals and populations have been influenced by power, knowledge, discipline and discourse; how these influences have changed over time and space; and how they are reproduced by the individuals and populations that they influence. Foucault's study of multiple historical periods, both geographically and chronologically, reveals the radical differences in the ways in which experience is ordered and realized.

Foucault followed a two-sided approach to historical analysis that he termed archaeology and genealogy. Archaeology refers to the process of investigating historical archives of various societies to discover the "discursive formations and events that have produced the fields of knowledge and discursive formations of different historical periods" (Danaher et al., 2000, ix). In other words, the archaeological element allows the researcher to pinpoint the various orders of discourse responsible for shaping the ways in which thoughts, ideas, propositions, and statements are articulated. In pinpointing these elements, we may then gain a more comprehensive understanding of the current thought processes, values, morals, and actions which flow through the discourse(s) of a particular society. Genealogy, on the other hand, is "a process of analysing and uncovering the historical relationship between truth, knowledge and power. Foucault suggests...that knowledge and truth are produced by struggles both between and within institutions, 
fields and disciplines and then presented as if they are eternal and universal" (Danaher et al., 2000, xi). While archaeology seeks to ascertain the principles of ordering and exclusion that enable discursive formations, genealogy focuses on revealing how truth and knowledge are tied to the ways in which power is exercised in societies.

The archaeological viewpoint provides a solid foundation for research objectives that concern discourses and discursive formations (and reformations) in societies. Foucault was particularly interested in the use of language, concentrating specifically on individual acts of language (discourse) as a gauge of language in use and knowledge in practice. Discourse shapes the way in which we view the world and ourselves; Foucault argues that the forces of discourse eliminate the possibility of the "self-governing subject," a term used here to denote a sovereign, autonomous self free from societal constraints. "Foucault rejects [the] idea of the self-governing subject, pointing out that what comes between ourselves and our experience is the grounds upon which we can act, speak and make sense of things" (Danaher et al., 2000, 31). Discourses become a substantive issue, then, as they serve as the funnel through which individuals' language, actions, behavior must pass in order to be legitimized. Individuals, Foucault states, are not originators of their actions or the makers of their own meanings. Instead, these actions and meanings are shaped by discursive and non-discursive forces that flow through the social positions that an individual occupies (Foucault, 1980).

The human body and its interaction with society have been examined by scholars who have addressed questions related to how bodies exist within society, because of society, and in spite of society. For Foucault, the body offered endless opportunity for historical analyses. Indeed, such analyses revealed the historical significance of the body 
in research, "Historians long ago began to write the history of the body. They have studied the body in the field of historical demography or pathology; they have considered it as the seat of needs and appetites, as the locus of physiological processes and metabolisms, as a target for the attacks of germs or viruses; they have shown to what extent historical processes were involved in what might seem to be the purely biological base of existence..." (Foucault, 1984, 173).

Foucault $(1984,173)$ references both politics and power relations in his conception of the body: "the body is...directly involved in a political field; power relations have an immediate hold upon it; they invest it, mark it, train it, torture it, force it to carry out tasks, to perform ceremonies, to emit signs." The body is bound up in its ability to both produce and consume, to perform as both object and subject: it is viewed as a "useful force" only if it is both a productive and a subjected body (Foucault, 1984). Foucault termed this the "body politic," which he conceptualized as "a set of material elements and techniques that serve as weapons, relays, communication routes, and supports for the power and knowledge relations that invest human bodies and subjugate them by turning them into objects of knowledge" (Foucault, 1984, 175-6). Here, Foucault highlights the consequences of bodies existing within society and society existing within bodies: the association (and interconnection) between object and subject, and the dual meaning of subject (subject to and subject as).

Foucault's analysis of the body and society reveals changes in governmental relations to both entire populations of bodies and individual bodies themselves. The realization that human beings may be trained and encouraged to work on themselves in order to shape themselves into useful, valuable resources for society came about with a 
change in the state's view of individual bodies. The state realized its vested interest in the health and productivity of each individual body that makes up society, as this productivity would determine the productivity of the state as a whole. This interest emerged alongside significant shifts in government around the eighteenth century associated with the European Enlightenment. The Enlightenment sought to "replace the old order of absolute sovereignty, injustice, ignorance and superstition with an order based on "enlightened reason and rationality"" (Danaher et al., 2000, 66). Prior to the seventeenth century, the state was conceptualized as a means to an end - the glory of the sovereign, the welfare of the people, and so on. From the seventeenth century onward, however, the strength, wealth and power of the state took priority; people were seen as resources, resources that had to be used and taken care of in an effort to strengthen the state (Danaher et al., 2000).

Governmental need to regulate, observe, assess, and categorize bodies emerged as an important type of social control and ordering. Individual bodies are valued as resources. Taken together, these individual bodies shape what came to be known as the "population." As a response to societal growth and the corresponding need for regulation, documentation, and control of the masses, the emergence of the term "population" was a significant addition to discourse. "The great eighteenth-century demographic upswing in Western Europe, the necessity for co-ordinating and integrating it into the apparatus of production and the urgency of controlling it with finer and more adequate power mechanisms cause population, with its numerical variables of space and chronology, longevity and health, to emerge not only as a problem but as an object of surveillance, analysis, intervention, modification, etc." (Foucault, 1980, 171). In an emerging effort to 
more specifically define the nature of an historical state, the history, geography, climate, and demography of a particular country were viewed as crucial elements in a new complex of power and knowledge (Dreyfus \& Rabinow, 1983). Once the government obtained concrete, specific and measurable information regarding its population, it could observe, track and control its citizens, thereby operating in a more effective manner. Study of the population came to be known as "political arithmetic," whereby effective government operation was possible through the ascertainment of the strength, or weakness, of its forces (Dreyfus \& Rabinow, 1983). Populations of individuals were observed, measured, trained, and tracked in order to increase the strength and durability of the state as a whole.

The body, as an object of power, is susceptible to the merging of political and biological forces. These forces, in turn, influence subjectivities, which are "put under the surveillance of authorities which safeguard the distinction between normal and pathological (perverse) in the interest of the continuation and purity of the Victorian family structure" (Gerhardt, 1989, 346). We see, then, how "the individual is reduced to a limited scope of autonomy carefully controlled within the "dialectics of social control"” (Gerhardt, 1989, 346) and how this individual is groomed for the betterment of the state. The future of populations - in terms of its strength and durability - was assumed by the state. "Once the politics of life [bio-power] was in place, then the life of these populations, and their destruction as well, became political choices. Since these populations were nothing more or less than what the 'state cares for for its own sake, the state was entitled to relocate them or to slaughter them, if it served the state's interest to do so"' (Dreyfus \& Rabinow, 1983, 138). Eugenics may be viewed essentially as "public 
health," as it was a measure enacted by states to better the health of populations. According to this line of thinking, genocide may be identified as a progressive reform movement (Engs, 2001). Kerr and Shakespeare (2002) trace sterilization, euthanasia and genocide as it has occurred historically and as it occurs presently. In Nazi Germany, for example, patients of "killing centers" were selected on grounds of "medical condition and level of productivity" (Kerr \& Shakespeare, 2002, 31). Dr. Leo Alexander provided a study of euthanasia during the Nuremberg trials, finding that over 275,000 were killed on the basis of the scientific orthodoxy of the early twentieth century, which materialized as prejudice against disabled people and racial minorities (Kerr \& Shakespeare, 2002, 44). The state decision to kill off populations of people was justified as a "service" to both society and the individuals themselves (Kerr \& Shakespeare, 2002, 45). The justification was that bodies and the larger population were subjected, used, transformed, and improved (Dreyfus \& Rabinow, 1983), thereby increasing the vitality of the state.

The necessity for entire populations and individual bodies to be guided and tracked, and thereby variably controlled, required a new form of power to emerge. For Foucault, the body is an object of power, which is produced in so that it may be controlled, identified and reproduced (Turner, 1984, 63). This power over the materiality of the body is called "bio-power." Bio-power, a power symbolized by life, replaced sovereign power, a power symbolized by death. Bio-power is the deployment of power over life; it is the "administration of bodies and the calculated management of life" (Foucault, 1984, 262). Through a dispersion of its various forces, bio-power appears to benefit those it affects. Further, this dispersion of its forces also allows bio-power to more or less seep unknowingly into a person's life. 
Bio-power affects us from two distinct poles of interconnected forces: discipline and subjugation of bodies and regulation and control of populations. One pole, the anatomo-politics of the human body, is centered on the individual body; specifically, on the body as a machine. This discipline focuses on the body's "disciplining, the optimization of its capabilities, the extortion of its forces, the parallel increase of its usefulness and its docility, its integration into systems of efficient and economic controls" (Foucault, 1984, 261-2). The second pole focuses on the regulation of the population as a whole, identifying each body with the mechanics of life, serving as the basis of biological processes (Foucault, 1984). Bodies are regulated in terms of propagation, birth and death rates, health levels, life expectancy, and the factors that cause each phenomenon to vary. The supervision of these processes is "effected through an entire series of interventions and regulatory controls: a bio-politics of the population" (Foucault, 1984, 262). This normalization and optimization of life occurs in a multifaceted, multi-dimensional manner such that rather than simply requiring the intervention of medical practice, health and disease were conceptualized as requiring multiple collective control measures (Foucault, 1980, 168-9).

Documentation and surveillance of bodies led to new responsibilities for the body. As Foucault states: "the 'body' - the body of individuals and the body of populations appears as the bearer of new variables, not merely as between the scarce and the numerous, the submissive and the restive, rich and poor, healthy and sick, strong and weak, but also as between the more or less utilizable, more or less amenable to profitable investment, those with greater or lesser prospects of survival, death and illness, and with more or less capacity for being usefully trained" (Foucault, 1980, 172). As the bearer of 
new variables, an individual's body is seen in particular ways (e.g., healthy or unhealthy) and is categorized based on this appearance (e.g., utilizable or futile). Each individual body is responsible for its status.

As medicine emerged as a form of regulation and control, it also gained popularity and success as a positive, life-giving force: "Once medicine gets a handle on how to cure disease (which really only happens in the 20th century, with the development of antibiotics, chemotherapies, the understanding of hormones and the ability to synthesize them, etc.), there is a shift in emphasis from death to life in medicine" (Hausman, 2006, para. 7). Attitudes toward life took on corresponding optimistic undertones as life expectancy increased, infant mortality declined, sickness gradually ceased to be endemic, and fatalistic attitudes toward death and disease were replaced by the notion that good health was within the reach of every citizen (Morantz, 1984).

The practice of medicine began as a domestic affair whereby lessons and practices were passed on from generation to generation. The professionalization of medicine carried with it the lesson that medicine is a specialized practice, one that is no longer appropriate in domestic environments. The professionalization of medicine meant corresponding secrecy and expertise within the field. Sickness and its alleviation, which once shared a relationship of "instinct and sensibility," free from social networks and experience, became privileged information. "It is this relationship, established without the mediation of knowledge, that is observed by the healthy man; and this observation itself is not an option for future knowledge...it is performed immediately and blindly" (Foucault, 1975, 55). Medical discourses emerged, having corresponding effects on health for both individual subjects and entire populations. 
With this shift, new strategies were created for the documentation and categorization of the health of the population. Health is based on normalization: while one individual is in a normal range for blood pressure, another may be within the normal range for cholesterol. Data from lab work, pap smears and temperatures are charted on statistical spreadsheets to compare an individual's results to the one defined as "normal." Populations of bodies are regulated by these numbers and statistics. How individuals live becomes an issue of regulation and control under the forces of bio-power, as it is through bio-power that the state is both concerned with and able to normalize and optimize life. The life of the individual is enveloped by the interest of the state. Thus, individuals come to realize that healthy, fit, strong individual bodies are the more valuable bodies, as they benefit the societal productivity at large. Health policy, for instance, has been cited as an example of biopolitics at work, as it ensures we are fit and well, thereby able to contribute to the workforce (Danaher et al., 2000, 125).

The regulation of health through daily health-related practices and concerns is not in itself a negative phenomenon. It is, however, a significant one. This regulation clearly illustrates the dispersion and corresponding control that bio-power has over our lives. We begin to understand how bio-power works through bodies. As Dreyfus and Rabinow (1983) indicate, the embodiment of bio-power is best understood through "micropractices": "To understand power in its materiality, its day to day operation, we must go to the level of the micropractices, the political technologies in which our practices are formed" (Dreyfus \& Rabinow, 1983, 185). These "micro-practices" in relation to health may consist of any number of cautionary measures taken to prevent illness. Bio-power is 
a regulatory technique; as such, it has a significant role in constituting a therapeutic regime.

Bio-power and its elements of personal responsibility, discipline and regulation were important preparatory steps, Foucault argues, for the introduction and eventual success of capitalism. Turner (1984) explores this relationship, highlighting the interest of the state in regulating individual bodies through a "variety of ideological apparatuses," stating that "The state has a very clear interest in preventive medicine for promoting health in populations which are rapidly ageing. Personal responsibility for health through exercise, diet and avoidance of drugs, reduces the tax drain of curative medical intervention. There is consequently an interesting alliance between the state, the medical profession and the healthy citizen" (Turner, 1984, 210). Turner talks about the "monogamous jogger" as the responsible citizen; this jogger may be conceptualized as having concern for both her own individual body as well as the body of the state. Conversely, the citizen who chooses not to jog may be deemed a burden to the state; her decision not to jog becomes an issue of moral deviance, a decision that equates to "selfinduced illness" (Turner, 1984).

In his theoretical work, Foucault investigates how society is embodied by entire populations and individual bodies. He does not view the body as a natural entity; rather, he conceptualizes bodies, and identities, as constructed through socially-contingent elements. Foucault criticized the "natural" or "biophysical" reality of the body which was the foundation of more traditional conceptualizations of the body. By examining the body through frameworks other than medical ones, Foucault developed a "more or less wholesale view of the body and disease as socially constructed entities: an ethereal matter 
in which the matter of bodies is no real matter at all, or perhaps more accurately, a discursive matter via the productive effects of power/knowledge" (Williams, 2003, 17). Terminology, a tool used to both reflect and construct a society, reveals how the definition, meaning and embodiment of a term such as "health" is heavily imbued with the goals and objectives of society. That is, how a society understands, visualizes, and lives health is understood as both product and producer of the norms, values, morals, and historical context of the society.

Foucault's theory of the social construction of bodies can be supplemented by the sociological analyses of Pierre Bourdieu (1930-2002). Bourdieu's contribution extends the understanding of how society is variably embodied and reproduced by individuals, a variation that is inextricably linked to an individual's social situation. The concepts of "habitus" and "cultural field" are principle components of Bourdieu's theory. Bourdieu interlocks these social phenomena to create a comprehensive view of social reproduction - that is, an individual's experience within and contribution to society. Bourdieu assumes that "the embodied actor is both indelibly shaped by, and an active reproducer of, society" (Shilling, 2005, 61). Or, succinctly and in his own words, "the body is in the social world but the social world is in the body" (Bourdieu, 1990). His theoretical framework is constituted by objective notions associated with structural analyses of the ordered body interlocked with the subjective notions associated with phenomenological writings on the body as subject (Shilling, 2005, 61).

Habitus is defined as "the ways in which we are produced as subjects through sets of dispositions - or habits - which predispose us to think and behave in ways that are adapted to the structures in which we are constituted. Since these are predispositions, 
they are embodied, durable and largely unconscious" (Danaher et al., 2000, xii). Habitus may be understood as a sort of "net" that contains, or guides, individuals' actions and practices. That is, though an individual is hypothetically capable of any number of actions, habitus defines, or tightens, the scope of the normative actions in which an individual will likely engage. The habitus is a "socially constituted system of durable, transposable dispositions which provides individuals with class-dependent, predisposed ways of categorizing and relating to both familiar and novel situations" (Shilling, 2005, 62). As Williams $(2003,30)$ states, "the habitus...is a 'structuring structure,' a 'socialized subjectivity' which gives rise to and serves as the classificatory basis for individual and collective practices. As such, it provides individuals with class-dependent, predisposed, yet seemingly 'naturalized' ways of thinking, feeling, acting and classifying the social world and their location within it." Habitus is a powerful and nearly indiscriminant force that sculpts an individual's experiences. It therefore becomes a significant component to an understanding of health in an individual's life.

Though habitus seems to have an unnoticed, nearly unconscious influence on individuals' thoughts and actions, Bourdieu is careful to refute the idea that individuals are incapable of agency. In fact, Bourdieu understands human agency as a necessary element for the persistence of social construction; this understanding distinguishes him somewhat from Foucault. In other words, Bourdieu recognizes that specificities within social construction both require and allow human agency in order to persist, “...while an agent might be predisposed to act in certain ways, the potentiality for innovation or creative action is never foreclosed: 'the [habitus] is an open system of dispositions that is constantly subjected to experiences, and therefore constantly affected by them in a way 
that either reinforces or modifies its structures"' (Bourdieu, as cited in McNay, 1999, 103). Habitus is generative in nature, recognizing a the relationship between the individual subject and the world as active and creative (McNay, 1999).

Bourdieu's theoretical concept of habitus represents a relatively limited, though still open, system of identification. As such, symbolic structure and subject agency relate to one another in terms of "regulated liberties" (McNay, 1999, 104). Bodies are not simply blank slates that absorb any and all social inscriptions. Rather, Bourdieu views the body as "the point of overlap between the physical, the symbolic and the sociological...The body is the threshold through which the subject's lived experience of the world is incorporated and realized and, as such, is neither pure object nor pure subject" (McNay, 1999, 98). Embodiment, then, represents that moment of negotiation, whereby the subject is constituted through dominant norms yet, simultaneously, is not reducible to them.

For example, the subject negotiates his or her ideas about health through classrelated micro-practices. The individual is endowed with the capacity for active decisionmaking and negotiation, “.... it is this notion of the individual being an actor who actively embodies, manipulates and changes discourses and makes them his or her own which is absent in Foucault. And it is an important absence, since it helps provide an understanding of how discourses change and develop, how some have become dominant while others fade away and how new discourses emerge" (Inglis, 1997, 17). In his theoretical work, Bourdieu highlights how individuals and society simultaneously produce one another. 
While the embodied individual is active in Bourdieu's conceptualization, this action is contingent on the relationship between the cultural field and habitus. The cultural field from which habitus emerges is also a significant consideration, as there are specific institutions, rules and resources linked to specific fields. As sites of cultural practice, cultural fields are fluid and dynamic entities constituted by "a series of institutions, rules, rituals, conventions, categories, designations and appointments which constitutes an objective hierarchy, and which produce and authorise certain discourses and activities" (Webb et al., 2002, xi). Fields are always changing, largely due to internal practices and politics, and by their convergence and interaction with other fields. In this way, both reproduction and transformation of an individual's position (and corresponding resources) are conceivable. An individual's habitus adjusts to reflect changes in position and access to resources. The cultural field of which an individual is a part plays a significant role in how this individual both makes sense of and embodies health discourses (Lupton, 1995).

There is an interdependent relationship between habitus and field; and the action of an individual reinforces norms within each cultural field. "The interaction between body and society involves a mutual determination which harnesses physical capacities and dispositions to the reproduction of the status quo" (62). People begin to act in ways that "make sense" within their cultural fields; an individual's behavior is more influenced by the desire to maintain the status quo than an outward prohibition from acting in a particular way.

Whether an individual decides to participate in health-related behaviors, and the specific behaviors this individual engages in, may also be tied to social capital, desire to 
obtain more social capital, social field, consumerism and the world of fashion. Social capital is a concept Bourdieu $(1986,248)$ developed to describe the social reproduction of social inequalities (such as social class). Bourdieu conceptualized this term as "the aggregate of the actual or potential resources which are linked to possession of a durable network of more or less institutionalized relationships of mutual acquaintance and recognition." Social capital is a characteristic of specific groups; as such, social capital "forces us to consider not only the existence of community social networks, but also the resources (potential or actual) possessed by the network and individual residents' abilities to draw upon the network for those resources in order to pursue a variety of goals" (Carpiano, 2006, 167). As social capital is intimately tied to social field, what is desirable in one field may be less desirable in another.

The relationship between class, health and lifestyles begins to bridge the gap between knowledge and action - how, for example, an individual defines the concept of health and how he or she then embodies it. Social factors influence the individual's knowledge about and participation in health-related practices: "games of different fields are never fair. The 'game of health' is clearly not on equal terms, but socially stratified depending on individuals' and families' relative access to, and embodiment of various forms of capital" (Meinert, 2004, 12). Whether an individual has access to health resources, such as health insurance, medical care, gym facilities, or nutritional counseling will be influenced, at least in part, by his or her capital. Activation of, access to and interest in health practices is also influenced by the individual's habitus, which produces varying levels of desire and perceived need for these resources. As an embodied, durable and largely unconscious predisposition to "think and behave in ways that are adapted to 
the structures in which we are constituted" (Danaher et al., xii), an individual's habitus may or may not involve the desire or perceived need for regular doctor visits, a low-fat diet, daily physical exercise, or vitamin consumption as components necessary to "be healthy."

The habits that people develop are the result, in part, of the cultural field and habitus in which people exist. The development of these habits is also tied to the social structure that makes it possible (or not) to go to the doctor, buy organic or low-fat foods and eat fresh vegetables and fruits. Bourdieu's concepts of habitus and cultural field bring in the element of individual agency, but also illustrate how our daily habits are structured by the knowledge of those with whom we are in close contact (e.g., parents, siblings, close friends) and the structure of social relations (e.g., whether we have access to health insurance and can afford to purchase healthy foods). Even if structural barriers to health are reduced or even eliminated, a person whose habitus does not involve the lesson that eating well and exercising regularly is not likely to value these activities as healthy or engage in these behaviors.

Culturally available health messages shape individual notions of what healthy means (for example, what health looks like and how health is enacted). The consumeroriented construction of these messages is an important component of this theoretical framework. Critical theorist Theodor Adorno (1903-1969) concentrated on capitalist culture in an effort to understand modernity. In his work, The Culture Industry (1991), Adorno argues that popular media, as the product of the culture industry, works to preserve the dominance of capitalism by keeping the population passively satisfied and politically apathetic (Gauntlett, 2006, para. 3). Mass media offer easy pleasures - illusory 
pleasures - which satisfy false needs created by culture rather than providing true happiness (Gauntlett, 2006, para. 5). The application of Adorno's theory to the health and fitness industry may be an insightful exercise. Adorno argues that popular culture creates and satisfies false needs to a passive audience. These false needs are satisfied by products, services, and commodities. Within the health and fitness world, Adorno may argue that individuals learn to use commodities to achieve the goal of health.

This commodification of the health and fitness world may affect an individual's conceptualization of what it means to be healthy, or how to live a healthy life. Although many people have the ability to take daily walks outside, the thought of "exercise" often conjures up images of the gym: spandex, treadmills, water bottles, weight machines, and sports drinks. Rather than walking, doing crunches or physical labor for exercise, the commodification of the fitness industry has equated the consumption of products as the way to become fit. Advertisements for treadmills, abdominal machines and "Nautilus" equipment attempt to sell these products as the image of fitness and, in turn, shape the way individuals think about exercise. These commodities may be associated with the word "exercise" almost instantaneously. Although an individual has the option to resist a second helping of dessert or to reduce his or her intake of fast food in order to lose weight, "dieting" or "nutritious eating" conjures up images of diet pills, diet programs (e.g., Slim-Fast, Weight Watchers, the South Beach diet), diet foods (e.g., "lite" ice cream, low-carb pasta, sugar-free candy, etc.). This connection between products and "obtaining" health may be more fully understood within a framework that allows for this critical assessment of mass media and commodification. 
Adorno's theory of the culture industry is significant to the analysis of various parts of the health industry. Albrecht (1999) examines the marketing of rehabilitation services and goods, cautioning that this marketing may be cause for alarm. As the rehabilitation business grows, Albrecht expresses concern with the benefactor of this growth. Rather than providing increased quality in services so that the consumer enjoys greater benefits, Albrecht proposes that the true benefactor is the company selling these goods and services. Capitalist drive for marketplace profit separates perceived need from actual need: "because of the complexity of the purchase decisions and the volume of information to be processed, patients often are not in control of their own health care, nor do they know whether the goods and services consumed are needed or cost beneficial" (Albrecht, 1999, 333). If market forces are in control of rehabilitation services, false needs for these goods and services may be created in order to increase profit (Albrecht, 1999). The result may be that consumers purchase products that are more or less superfluous to their medical needs. Despite increased purchasing of products, however, the patient's quality of life may or may not increase. Further, patients learn that additional product purchase is the key to a better, more comfortable way of life regardless of the actual patient benefit.

Gender is an important consideration in the analysis of any social phenomenon (Lorber, 1997). In contemporary America, health promotional messages ingrain the notion of women as health caregivers (Lupton, 1995), which gives them a certain, crucial role in the health of America. Simply put, health in contemporary consumer-oriented American society is experienced differently depending, in part, upon gender (Ratcliff, 2002; Lorber, 1997; Freund \& McGuire, 1991). Gender, as a "ubiquitous system of 
oppression," influences and is influenced by the organization of society, including women's health, health research and health care (Ratcliff, 2002, 7). Women's health may be compromised by their status in contemporary American society, as poverty, lack of power, racial and gender discrimination, and the gendered division of labor all affect how healthy women are (Ratcliff, 2002). The symptoms, pains and weaknesses that are attributed to illnesses, the risks and protections against illnesses, the responses that are elicited by health professionals to illness, and the priority of treatment, research and financing are influenced by gender, in conjunction with other socially constructed categories such as race and class (Lorber, 1997). The social constructions of both health and illness must be gendered because "gender is one of the most important statuses in any society" (Lorber, 1997, 5).

Gender plays a role in shaping the experience of "health": Men and women have different life expectancies (women live longer); men have more life-threatening illnesses than women, yet women become ill more frequently; men are more likely to than women to engage in high-risk behavior (Freund \& McGuire, 1991, 27). Within the health care system, female patients have less power and less satisfying communication than male patients (Ratcliff, 2002, 7). Women tend to adopt more healthy lifestyles than men in terms of frequency in wearing seat belts, getting adequate amounts of sleep and exercise, eating a healthy diet, and managing stress (Lorber, 1997). On a daily basis, then, the experiences of women and men in terms of health and health care are noticeably different.

The ways in which women conceptualize and embody health may be quite different from the ways in which men conceptualize and embody health. Cultural 
messages related to health and health promotion target men and women differently (Lupton, 1995). Critics of health promotion messages point out the routine reproduction of stereotypes in advertisements, including: the portrayal of women as passive, vulnerable, sexually available, youthful and beautiful (Lupton, 1995, 45). Health promotion campaigns oftentimes appeal directly to women's anxiety about their bodies, appearance, attractiveness, and weight (Lupton, 1995). Contemporary American society tends to evaluate women based on their appearance and men based on their performance (e.g. occupational) (Freund \& McGuire, 1991). Consequently, women have learned that appearance is the way through which they may exhibit "mastery and achievement to others and to herself" (Freund \& McGuire, 1991, 51). The "ideal" bodily appearance is firm and toned, as fat and flabby bodies are indications of a lack of self-control, unregulated behavior, and unrestrained hunger (Bordo, 1993). Bodies with excess flab and flesh are targeted by ads for diet products and weight loss programs aimed at getting rid of the "enemy," with copy reading, "Now...get rid of those embarrassing bumps, bulges, large stomach, flabby breasts and buttocks. Feel younger, and help prevent cellulite build-up" (Bordo, 1993, 189). Women are encouraged by cultural pressures to mold their bodies into firm, toned, lean shapes and, in turn, are told they will be accepted as more visually pleasing, attractive and healthy (Bordo, 1993).

The work of feminist scholar Patricia Hill Collins is an important addition to this theoretical framework. The focus of Collins' theoretical work is the development of a Black feminist thought that provides a viewpoint for African-American women created by African-American women. This viewpoint, then, reflects the social realities, experiences, pasts, presents, and futures of African-American women in an accurate, 
substantive, and meaningful way - in a way that is arguably the opposite of the oppressive, limiting, and binary viewpoint imposed upon Black females by elitist White males.

Collins developed the concept "matrix of domination" to describe the overall social organization within which intersecting oppressions originate, develop, and are contained. With a depiction of the complex hierarchies actually shaping society, Collins argues that we are able to see how race, gender, class, citizenship status, sexuality, and age shape any group's social location in the transnational matrix of domination. Collins constructed the matrix of domination to replace "additive" models, which understand oppression and domination in terms of an either/or dichotomy. In additive models, a person is either White or Black and is then ranked based on his or her category. Alternatively, the matrix of domination takes a both/and conceptual stance. This understanding recognizes the privilege and penalty that all groups face in a historically created system. White women, for example, are privileged by their race but penalized by their gender.

Collins' matrix of domination allows for the consideration of the ways in which class, race and gender come together with varying effects on women of different races and classes. Race intersects with class and gender in such a way that women of varying classes, races, levels of physical ability, levels of education, occupation, etc. encounter society as a matrix of variables that either dominate them or don't. In the exploration of health concepts and health behaviors, it is important to consider how race, class, gender, and other social variables influence the life experiences of each individual. An individual's notion of health and his or her participation in health behaviors are 
constructed within varying levels of oppression. The privilege and penalty that a woman encounters based on various societal variables may shape her experiences of health, and Collins work allows me to explore this important intersection.

Summary

The theoretical concepts put forth by Foucault, Bourdieu, Adorno, and feminist scholars Susan Bordo and Patricia Hill Collins offer a relevant, comprehensive theoretical framework for the examination of women's health concepts and health-related behaviors. The construction of health concepts and the embodiment of health are shaped by both contemporary capitalist society and the reproduction of class, field and habitus.

Foucault's notion of bio-power suggests that regulation and control of bodies and subjectivities are dispersed throughout contemporary society. Healthy, productive bodies are more valuable to capitalist societies, as they are better able to participate in the work force and are thereby able to consume. Bourdieu's notions of cultural field and habitus suggest that the construction of health concepts and health behaviors are shaped by social environment, including class, familial values, and education. An individual's cultural field provides a conceptual "net" within which norms (in terms of behaviors, values, and thoughts) develop for an individual. Habitus is constituted by the patterns of behaviors, values and thoughts that an individual then embodies. Health concepts and health behaviors are shaped in part by these factors. Adorno offers a critical look at capitalist consumer culture, suggesting an analysis of the production and consumption of health and fitness as commodities. Products are sold based on promises to consumers to provide certain outcomes ("toned" arms, "sculpted" abs, flab-free stomachs). The consumption of 
these products suggests the experience of health and fitness as negotiated within consumer culture. Feminist perspectives on how health messages, health care, and health research are produced in contemporary society are critical to understanding the different health-related experiences (and, therefore, conceptions) of men and women. The intersection of race, class and gender creates varying levels of privilege and penalty for individuals as they encounter society. This oppression will, in turn, effect the health experiences of the individual, a reality which Collins' matrix of domination helps me to understand. This theoretical framework provides a basis for the exploration of health concepts; more specifically, the relationship between cultural messages of health and the definitions and concepts of health that are embodied by women, and how these concepts are shaped by capitalist culture, habitus, field, consumer culture, and feminine cultural ideals. 


\section{METHODS \& SAMPLE}

I conducted a qualitative interview-based study of a sample of self-identified healthy women to examine how they define health and enact health through behavior. I obtained my sample of twenty-five women who self-identify as healthy through nonrandom convenience sampling methods. I used grounded theory to analyze my data through ongoing data collection and simultaneous data analysis and theory building. I focused only on women in my research in an effort to fill the void within sociological research that has left the construction of health concepts and health behaviors of women in North America largely unexplored. Contemporary American society has linked aesthetics with health, resulting in a dominant image of thin, toned and firm bodies as healthy, desirable ones. Further, many health promotional messages target women as the facilitators and regulators of healthy living, a responsibility which is enacted through their roles as wives and mothers. Women are consequently given a crucial role in the health of contemporary America.

I conducted in-depth, semi-structured personal interviews in order to examine women's conceptualizations of health. The interviewing process took roughly four weeks to complete (March to April, 2006). The majority of interviews took place on the University of Louisville campus in one of two locations: in Lutz Hall or the University Club building. In four instances, however, interviews took place elsewhere, in a mutually agreed upon location. Qualitative methodology was chosen as an appropriate approach to 
explore health concepts and health behaviors because I wanted the ability to explore conceptualizations and connection in-depth with each study participant. I used nonrandom convenience sampling to acquire participants for my sample, which is accepted as legitimate in the field of sociology (Denzin \& Lincoln, 1994). Convenience sampling was the most effective techniques for my study, as it allowed me to recruit informants in the area I was studying and because representativeness is not a goal in exploratory studies. I also used snowball sampling to recruit participants, whereby participants recruited other individuals who they knew to be eligible for the study.

My sample includes twenty-five women, each of whom was over the age of 18 and who self-identified as healthy. In response to the advertisement for the study (see Appendix A), many individuals stated reasons why she thought herself to be healthy along with requests for confirmation from the researcher that she was indeed "healthy." Care was taken to neither confirm nor disconfirm this individual's hesitation. Rather, the criterion was simply restated to ensure that the ultimate decision for participation - and, consequently, self-proclaimed health - was left up to the individual. I was then able to examine the similarities and differences among the reasons why individuals identify themselves as healthy and the gap between definitions of health and participation in health-related practices from this specific sample of women.

The study received IRB approval prior to being conducted. Recruitment flyers were posted on campus in three specific, IRB-approved locations (the arts building, humanities and sociology building, and a gym facility). The contents of the recruitment flyer (see Appendix A) were also approved by the IRB. Every participant signed an informed consent document before the interview took place and each was asked for 
permission to be audio-taped; only one participant chose not to be audio-taped. The interview schedule (see Appendix B) was pilot-tested in an attempt to eliminate ambiguity or problematic parts within its contents prior to conducting interviews. I encouraged the participants to answer questions in full, which I elicited via probes as appropriate. I also encouraged participants to add information that was not covered in the interview schedule. Upon completion of the interview, participants were compensated (\$16) for their time and inconvenience.

The semi-structured interview schedule was developed based on previous studies that have investigated concepts of health (Hodgins, 2005; Manderbacka, 1998; Barrett, 1995). The schedule began with questions pertaining to the respondent's demographic background. The questions that followed were related to "overall health" and healthrelated behaviors. The section began with a question which asked the participant to describe why she self-identifies as healthy. Participants' answers were often long and detailed. Additional questions related to health invited more discussion of the dimensions of health. Thus, the section included broad health-related questions as well as more specific ones in order to assess this topic from a variety of angles. This section was typically the one which respondents spent the most time mulling over in order to consider their stances, seemingly putting these specific thoughts together for the first time.

A section with fitness-related questions followed, in which individuals were asked to talk about their participation in fitness, for example, how often they exercise, what they do for exercise, what they define as exercise, hesitations or obstacles to participating in exercise (such as discomfort or dissatisfaction in exercising at the gym), and sources for fitness-related information. This section invited answers that eventually led to an 
indication of existing patterns, such as the preference for physical activity that was outdoors and low-impact. Finally, I posed nutrition-related questions. These questions revolved around the participant's daily diet, her definition of a healthy diet, what advice she would give others on improving their own diet, and the sources from which she pulls for nutrition-related information.

Interview data were analyzed using a modified version of the grounded theory method. Grounded theory is a general methodology for developing theory that is grounded in data that was systematically gathered and analyzed (Strauss \& Corbin, 1994). As the primary goal of this research was to gain insight into dimensions that constitute women's conceptualizations of health, grounded theory provided an appropriate data analysis schema in which successive iterations of data collecting, analysis, and further collection result in thick analytic themes and categories. The methodology of grounded theory involves the generation of theory and doing social research as two parts of the same process. Grounded theory methodology has redefined the usual scientific canons for the purposes of studying human behavior in its suggestion that credibility of theory be based on the "detailed elements of the actual strategies used for collecting, coding, analyzing, and presenting data when generating theory, and on the way in which people read the theory" (Glaser \& Strauss, 1967, 224).

The new data I collected served to either strengthen or weaken the theory I was building throughout this research process. I transcribed each interview after it was completed and read the transcription of each interview several times, which informed the following interview both in how it was conducted and how it was interpreted. I tested the concepts I detected as emerging from the data against new data as it was obtained, which 
is an important process in increasing the validity of research findings. Further, I engaged in brief journaling following interviews to capture important themes that emerged throughout the interview process.

I transcribed each audio-taped interview in full. The interview that was not audiotaped was documented via note-taking throughout the interview; these notes were then recorded electronically for data analysis purposes. Transcriptions were carefully read multiple times to allow me to gain a greater sense of familiarity with the content. This allowed themes and patterns within the data to emerge. I cut and pasted related statements, quotes, and chunks of text from the transcriptions into separate documents. I was then able to create thematic documents to describe and organize the data. The thematic documents contained patterns that I detected throughout the data analysis, which I labeled accordingly (e.g., health as an achievement and health as balance). After reviewing these documents, I found patterns within participants' accounts of health concepts and health behaviors. These documents were separated and continuously modified as my analysis of each interview allowed the themes to emerge.

\section{Demographics of sample}

The population consisted of women who were over the age of 18 and considered themselves to be healthy. Twenty-three participants described themselves as White or Caucasian; one described herself as Indian; and one as Puerto Rican. While participants' ages ranged from 19-66, most fell within the age range of 19-24 with a median of approximately 28 years. Marital status was spread across the board, though not evenly: 16 participants were single, five were married, three engaged, and one divorced. 
Household income, defined as the income of the household in which the individual grew up, was varied: nine household incomes were estimated within the $\$ 20,000-\$ 60,000$ range; ten household incomes were estimated within the $\$ 61,000+$ range, with four over $\$ 130,000$. For six participants, household income was not documented, due to either uncertainty or unwillingness on the participants' behalf. Religiosity and spirituality were also varied: nine participants described themselves as identifying moderately to strongly with a religion; five identified loosely; seven had no preference; and one identified herself as agnostic. Twenty participants held jobs ( 12 of which are part-time jobs), four did not work and one was retired. Eighteen participants attended college or graduate school (13 were enrolled as full-time students). Three participants did not have health insurance at the time of the study. The remaining 22 participants had some form of health insurance, typically coming from parents; however, three participants pay for insurance themselves. 


\section{FINDINGS \& ANALYSIS}

I analyzed twenty-five documented accounts of health concepts and health behavior in order to examine how women conceptualize these concepts. The women in my sample described their concepts of health as an ongoing, negotiated process. Health was an achievement that recognized wider cultural messages of health, yet was based within the individual's social situation. The women actively protected themselves from allowing cultural notions of health to dictate their lives. Larger cultural messages of health were translated into messages that reflected her habitus, thereby shaping her personal ideas about health. Both structural influences and individual agency are involved in the process of constructing health concepts.

An important interrelationship between human agency and social structure in the construction of health concepts and the embodiment of health emerged from the data. Health identity, a concept I developed from an analysis of these documented health accounts, captures this relationship between agency and structure. Health identity may be described as the translation from cultural and social messages of health to an individual's health conceptions and health behaviors on a daily, conscious level. This translation is an important element of health identity, as it acknowledges socio-cultural influences while also acknowledging the role of the individual, who actively constructs an alternative to cultural and medical views of "health" that are perceived as unattainable. Furthermore, this translation personalizes the project of health, thereby becoming a committed, 
valuable and worthwhile investment of time and energy. Health is something that "fits into" a person's daily routine rather than developing as something separate from it. In this sense, health is a negotiated, achievement-based component of identity that involves negotiating positive alternatives to unattainable cultural messages using individual preference and voice.

The findings suggest that "health" is achieved through a daily, negotiated balance of multiple dimensions. Interview questions and probes about "being healthy" and "feeling healthy" allowed multidimensional conceptions of health to emerge. Individuals discussed various dimensions in their definitions of health, including: diet, exercise, stress reduction, mental health, happiness, self-respect, self-esteem, spirituality or religiosity, rarity of illness, healthy weight, normal medical tests (e.g. cholesterol, blood pressure), abstinence from smoking, abstinence from or moderation in drinking, social activity, familial closeness and contact, and education. The women in my sample described their personal achievement of health through attentive, conscious and consistent effort. Some individuals valued certain health practices (e.g., eating nutritiously) over others (e.g., exercising daily). The variation in terms of each individual's specific health-related concerns may be understood as the consequence of differences in the habitus of each individual.

Health was described by participants as a "personal project," which she negotiated by translating cultural messages of health into messages that were more fitting to her daily routines, habits, preferences, and patterns. Personalizing health concepts was an important component of these women's health concepts, as it allowed the goal of health to become individualized and pertinent. With this personalization came the notion of 
personal responsibility for health. Rather than assuming the state or community was responsible for maintaining her health, interviewees discussed health as a goal to which she felt very connected and very responsible.

The women spoke of feeling encouraged and motivated to regulate their behaviors in an attempt to mold them into forms that resembled a healthy lifestyle - namely, eating healthy meals, getting regular exercise, relieving stress, and getting an adequate amount of sleep. Health promotion logic influenced the ways in which the women described health, namely in feelings of self-responsibility and self-surveillance. And yet, the influence of promotional messages did not determine the individual's daily practices. The women spoke clearly about their desire to fit health into their lives rather than adopting a lifestyle that required foreign or unnatural practices, such as working out four hours a day or never enjoying a slice of chocolate cake. The promotional message to be autonomous seemed to encourage these women to insert health into their lives rather than inserting themselves into the cultural ideal of health.

Participants described the process of "being healthy" as a realizable alternative to unachievable body ideals. The process of "being healthy," then, meant that participants resisted an uncritical acceptance of cultural bodily ideals. Health concepts are the outcome of a negotiation process: as individuals consume culturally-available health concepts, they translate them into their lives based largely upon their habitus. These ideals shape ideas about health as a balanced, realistic, conscious, give-and-take negotiation. Health was typically envisioned first as a physical well-being that was not necessarily a certain shape (slim, muscular and toned) but, rather, was the result of eating 
healthily, exercising on a regular basis, getting enough sleep, keeping stressors at a minimum. Health was a feeling rather than a particular cultural aesthetic.

An absence, or rarity, of illness was important to most women's concepts of health, yet was not the sole component. Health was defined as a project into which an individual may invest varying amounts of consciousness, time and energy. In this way, health could be achieved even after diagnoses such as Lupus and chronic fatigue syndrome, breast cancer, and heart disease. Health was also very typically described as a personal responsibility: the women assumed responsibility for making healthy food choices, exercising, getting adequate amounts of sleep, relieving stress, abstaining from smoking and excessive drinking, and so on. Women also expressed concern for the health of others, criticizing poor eating habits of close friends, fiancées, boyfriends, and family members. While being healthy was a priority for these women, the project of health was not something they let overwhelm their thoughts and actions. Indeed, preventing the project of health from dominating their lives was an important component of health itself.

Balance and moderation

Almost every interviewee mentioned the idea of "balance" in some form or another within her discussion of health. At times, the balance was mentioned in direct relation to diet; at other times, it was mentioned in terms of exercise. Balance was also mentioned in terms of achieving health. This achievement required conscious, daily attention to various components of health - such as diet, exercise, time management, and stress reduction. Balance was expressed in a positive, harmonious accomplishment. 
Balance was discussed literally, as a means to incorporate food from all the food groups, for example; hypothetically, in terms of the negotiation of ups and downs, successes and failures in the project of health; and holistically, in terms of considering all identified facets of health, including mental, physical, spiritual and emotional. Many interviewees were concerned with maintaining positive connotations of "health," which was achievable if the individual's concept of health was flexible. Health was not discussed as a rigid set of rules. Rather, health was discussed alongside balance, flexibility and happiness. The women discussed the importance of allowing for fun, letting loose, enjoying desserts or enjoying time off from exercising. Indeed, this flexibility prevented the individual from pushing too much, not enjoying herself, or feeling too constrained by rigidity.

(Note: Boldface type indicates my questions and probes.)
“...being in Europe, I never exercised but we did walk a ton - like my friend wore a pedometer and we were getting like 25,000 steps a day, so that's exercise - I think of that as exercise - balanced out all the beer and cheese..." (C12; age 24).
"[A healthy diet is] balanced...from all of the food groups. Um, low in fat. Balanced" (Ok, so a diet that includes elements from all of the food groups?) "Yeah - including the ones that are tasty but obviously...in small doses. Just because if you can't have a little tasty goodness every once in a while, what good is livin'?" (C3; age 23).

Balance is negotiated within the context of life. When individuals discussed their health behaviors, many discussed practical limitations of time, money, and energy. A balanced view of health, then, involved flexibility in terms of personal expectations; if 
her expectations were not met that day or that week, for example, the participant

understood this as an element of being healthy. The notion was that although she could be doing more, she was doing an amount that "worked" for her and that made her feel good. Participants were aware that, yes, there is more that could be done but I do not want to and do not necessarily see a reason for that change in order to be healthy. One interviewee emphasized her contentedness with the daily activity that she did fit in, a contentedness she contrasted with the obsessive nature of her former roommate:

"It's honestly that I've just set her at a bar that I, that I'm like: I don't reach that bar. I mean, she doesn't just exercise because she wants to look, like fit. She embodies, oh, when I exercise I know that that is good for my body and I know that I am probably preventing diseases, or - years from now, I'll have strong bones. And I mean, I don't even think about that. Um, but I think I put her at a bar that, I feel like it's kind of a pedestal but it's not my goal to join her on that pedestal because I don't really...her mindset is not the way I want to be thinking either. Like, I want to find some sort of medium between like, "I'm so relaxed about exercise that I never get around to it" and "I'm so, it's so important to me that if I don't do it for a day, I feel stressed out," (C13; age 27).

As another interviewee discussed her fitness regimen, she stated that because she was unhappy with her father's expectations, she stopped running with him. It was only after she began her own, more relaxed regimen that she was able to say she enjoyed running:

"I had stopped running for a little while, but and that, like my goal was just to get back to running and stuff. Like, I'm not the type of person who can go run 5, 6 miles. That was one of my goals was to 
be able to run a mile and then walk and stuff like that, not to be like completely exhausted when I got through working out and to be able to do other stuff afterwards (why did you stop running?) Um, because my dad made it not fun (ok, so it was like, I don't want to do this?) Yeah. Like I ran track in high school and then, cause like when I first got to college, I didn't really, I didn't really want to run" (C14; age 19).

Many interviewees rejected the idea of obsession with health in their own life and criticized this characteristic when it was present in the lives of others. Obsession with dietary habits and physical activity was unappealing to the majority of interviewees, which reiterated the notion that participants' activity and concern for their health was consciously held steady at a balanced level. In other words, while participants acknowledge the value of certain health-related activities, they were active in limiting their level of participation in these activities. The idea of moderation structured the discussion of an individual's ability to participate in health-related behaviors in a reasonable, "non-obsessive" way. For example, many interviewees defined a healthy diet as one that incorporated all foods, but in moderation when possible:

"I do include stuff from all food groups, I tend to be more favorable towards dairy, you know, I like cheese and yogurt. I really include probably more than 3 recommended servings of those. Um, and lots of fruits and vegetables - salads...Everything in moderation" (C6; age 28).

"Balanced proteins, whole grains, leafy greens, I eat a ton of vegetables and beans and certainly also peanut butter and jelly and integrating those things that are stereotypically healthy, that you see in the new food pyramid, but then also of course the things that I really like -but you know, everything 
in moderation. And sometimes not in moderation and I figure that's just all part of the flow" (C25; age 24).

Participants defined "obsessive" versus "non-obsessive" behaviors by assessing the health-related behaviors of others. Rigid rules in terms of eating and exercising were frowned upon and often categorized as "obsessive" by the participants. Keeping these undesirable behaviors in mind, participants formulated less rigid, more relaxed health behaviors. Several interviewees discussed this process of finding a balance in terms of participation in health-related behaviors:

(Who exemplifies health in your opinion?) "My dad. (How is he healthy?) Um, he's really big on not eating close to when you go to bed and he runs every day and works out 4 times a week and he's, he's just like a health freak (Is he mostly just physically active, or does he focus on food too?) Yeah, he eats good too. (Do you model your lifestyle after his?) Well, he affected a lot of what I do - like, that's why I started running and stuff. Like my dad, he kind of made me get into the running thing; I mean, he didn't make me, but he I guess influenced me to do it and stuff... (Do you feel like you will ever be as healthy as he is?) No. (Do you want to be?) No - I think he takes it to a different level"' (C14; age 19).

(Do you ever compare your own body to others'?) "Oh sure - I think we all do that. I guess I can't say that, but sure, I do. Sure, it's always a little, I've got a: "They can do that, I can do that!" (So are you like, I should be able to do that?) No... (Is it like an admiration?) Well, I guess sometimes when you do it by yourself, you are soft - easy on yourself, so you think if they can do that, I can do that. But I just try to maintain a balance. So...I mean, my daughter could spend hours and hours and hours - I mean, nothing else gets done in 
her life because she spends all her time exercising and working, and I think that's too much. But, I might as well be talking to the wall" ( $\mathrm{C} 24$; age 66$)$.

Individuals expressed an interest in finding physical activity that was enjoyable.

The feelings of happiness and enjoyment in her routine appeared to serve as an indication that she had successfully achieved that sought-after balance. While she was participating in healthy activities, she was doing so in a way that allowed her to find activities that made her happy and that allowed her to participate in these activities on a feasible and realistic level. Health, then, fit into her life and was experienced as personalized. In terms of physical activity, one participant reflected on the importance of staying in tune with what makes her happy as she makes decisions related to physical fitness; for her, fun activities allowed her to maintain a relaxed attitude toward fitness:

"I love to rollerblade, hike, we - my family and Igo camping a lot, my boyfriend and I are always doing something athletic. Swimming -I have a pool at my house too, so over the summer, I swim every day. I'm like a fish. Yeah, just things like that. I like to do fun things. You probably won't see me at the $\mathrm{Y}$ on the treadmill, counting calories. Just kind of, yeah, just anything athletic...fun things, I like to bike...just things like that, and like I said, camping - you do all kinds of things outdoors and it's a good workout" (C10; age 20).

One individual made a conscious effort to have fun while she ran, and tried to communicate this message to others. In fact, this enjoyment was a necessary in defining both herself and others as "healthy":

"For years, I didn't like running because I thought all runners looked miserable when they're doing it whereas other people who are biking or 
rollerblading or walking or playing with their dogs, they're enjoying it more where runners look like they're about to die and it just looks like it sucks. So when I first started running, I thought, oh this sucks, everybody hates running....And then I thought, no it's really nice! So then I started consciously smiling at everybody so they would know that I was running and having a good time and that I really liked it. So, one part of it too, so if somebody is really fast and looks like they are a really great runner but looks just miserable, I don't look at him and say, oh he has a great body, I say, oh - that's too bad! He doesn't have to be so fit and could really have a great time...don't do it if it's that bad! That's not healthy either!" (C25; age 24).

Another theme within the notion of balance was "effort toward" or "trying to" achieve health, or engage in certain activities that she associated with health and healthy lifestyles. The realization was that, although other responsibilities or commitments interfere, the individual is aware of her desire to be healthy and implements this project to the best of her ability. In this way, health becomes a feasible and realistic process rather than a rigid regimen of rules:

"So I feel like I try to exercise and I try to eat healthy and I also try to get enough sleep - I identify myself as healthy because I try at these things - they don't always happen, but I do think that they're important" (C8; age 19).

"Mainly, especially now, in the past few years, as I've gotten older, it's like, what can I get in that I can incorporate into my daily life that will still keep me healthy and it's not going to stress me out to the point of - "Oh! I didn't go to the gym today" or "I don't feel like driving across town to go to the gym..." You know, so I think it's about finding that balance, that's kind of - more of that now. And you know, I like to play basketball, so if I go and play 
basketball, Great! That's my exercise for the day. Now, sometimes I might do that and go to the gym, but as long as I do something, that's kind of my goal .... as long as I'm doing something! You know, playing a short game of tennis with my friend..." (C7; age 24).

And finally, balance was enacted in terms of "all things considered." In this way, study participants conceptualized health as multidimensional and, in attempting to pursue health, she deliberately concentrated on more than one dimension of health in order to feel her "healthiest." Health was a holistic concept:

"In a day, if I go to class and I feel like I've learned something and I do some yoga and I have a good meal and I listen to some music...then I feel healthy. I feel good" (C1; age 21).

"...health, for me, is a lot of different things and I think it comes from the yoga idea that, and it's so cliché - you hear it all the time - but body, mind and spirit. Yoga is, the word yoga means union - to pull together all those pieces, and that they're not separate - it's not like there's my physical self, and then over here's my mental self and then my spiritual self - they integrate and the more that they integrate, the more health you have on all of those different levels because you don't feel like those pieces, you know you feel like you're one whole thing and to me, you see everything as spiritual even the food that I eat, the exercise that I do, the connections that I make with people, and so that part sort of permeates all the different interactions, at least, as I try to do" (C9; age 29).

While health was described as a negotiated balance, this negotiation was oftentimes realistic in terms of time, energy, and financial constraints; loose and flexible enough to "fit" her life and the desire to "splurge"; and conscientious of satisfying all 
facets of health deemed important within a multidimensional understanding of health. In this way, the notion of health as a negotiated balance was reflected in participants' expectations for participating in healthy behaviors; while several dimensions were present, so too was the understanding that not all dimensions would be (or needed to be) satisfied every single day.

Health as an achievement

Participants expressed health as an achievement, which required daily conscious effort, self-regulation, self-surveillance and self-responsibility. Interviewees described investments of time, energy and finances into their projects of health. The notion of preventing illness, preventing problems and "doing everything I can" to remain healthy was evident in many interviews. Maintaining health was discussed as a constant concern, as something which a person must work at, pay attention to and take responsibility for. One individual in particular was especially aware of staying focused on her health because she did not have health insurance:

"Well, I don't have health insurance, so I need to do all that I can to keep myself from getting sick or from being, you know I think to a certain extent, your immune system can be suppressed by outside factors that makes you even more susceptible to disease - like stress, absolutely, - lack of sleep, smoking, drinking - to, in excess - but a lot of it, I think is stress, sleeping habits, eating habits. And, just pretty much that. And eating habits too. If you're eating unhealthy, then, um, it doesn't really set your body up well to do what it needs to do...I reexamined my own -I guess the control I have over my health. You know, and, just because it's easy to assume that you have no control, but I think 
that you have a good deal of control over the shape that you're in on a daily basis" (C17; age 24).

When interviewees discussed times in which they were not as focused on their health or were not able to devote as much time, energy and efforts into their project of health as they would have liked, they described unhealthy feelings as the result. This period of inattentiveness to her health was marked by discontent and disappointment. Health was identified as a daily achievement requiring daily health-related behaviors.

"I just want to be fit and you know; and when I don't do the things I know I need to do, then I know that I'm selling myself short because I need to do those things... (So you feel there are certain things you need to be doing to even hope to achieve...) Right, right - like I can't sit in the chair every day, 7 days a week, and then expect that this is going to happen" (C19; age 55$)$.

“...the week before spring break, that was a horrible week, because you know, stressful tests and things and it's just, my health gets put out of my mind when that happens, then it goes downhill" (C8; age 19).

Health was discussed as an achievable possibility, even in the face of diagnoses, illnesses and surgeries. Under Armstrong's (1995) notion of Surveillance Medicine and Frank's (1995) notion of the remission society, health can be reclaimed. Health exists alongside illness and health can exist despite illness. According to my study participants, health is an everyday, conscious achievement and is, therefore, possible for everyone. One interviewee, who was diagnosed with Lupus, an autoimmune disease, took daily 
medication to prevent recurrence, but was consciously aware of her daily activities in hopes of keeping herself in remission:

\begin{abstract}
"As far as I'm concerned, I' $m$ in remission - I take an anti-malarial drug that is supposed to quell the symptoms but I, my lab work has been excellent for the past 2 years... I have to be more aware of how I treat myself and the amount of sleep that I get and photosensitivity, which is sensitivity to sunlight is also a big factor - so, going out to the beach is fine but I have super careful that I put on sunscreen, so I put on sunscreen so I don't end up sick..." (C11; age 19).
\end{abstract}

One interviewee was diagnosed with chronic fatigue syndrome at an early age, and yet, in taking daily measures and monitoring her lifestyle in order to keep this illness in remission, she defined herself as a healthy female:
“...when it went into remission and I was able to start leading a more normal life, then um, I just made sure that I stuck to what the doctors told me... as far as routine and food and sleep and all of that so that I could prevent it from popping up again in a bad way....although I manage it and it doesn't keep me from doing things, I worry about that coming back - leaving remission and coming back, so I do things to make sure that doesn't happen that I've learned in the last 15 years how to do. (Like getting enough sleep?) And having vitamins and having a routine. Eating right. (So those things are the most important to you?) Definitely" (C2; age 30).

Another interviewee suffered from two very severe bouts of strep throat. The second bout was such a shock to her body that she developed hypoglycemia. Rather than knocking her into an "unhealthy" category, her hypoglycemia, as well as the threat of 
recurrence of severe strep served as reminders to engage in the activities and choices that made her the healthy person she believed herself to be:

\begin{abstract}
"Well, over the last three...since I had strep throat, I had a lot of, I mean it really changed my body and I suffered from hypoglycemia for a little while, and immediately sought out ways to try to regulate that, and learned all about the glycemic index and over the last three and a half years, I feel like I've read every health book, every internet site that's available, you know trying to figure that out...And so you know, I tried to get myself back in alignment sort of with my own personal diagnostic" (C20; age 37).
\end{abstract}

One interviewee was diagnosed with breast cancer and went through the process of reclaiming her health after the diagnosis:
"... and then of course having the breast cancer a couple of years ago. I mean, you know you're not going to live forever but you want to live, you don't want to live poorly, you don't want to live being sick. So, you know, you get maybe a little more serious that you're not going to let that happen - you start looking out for what's best for you -it's more of a gradual (a progression?) Yeah, exactly. And, you know, now I have the time to do it, so..." (C24; age 66).

Preventive, precautionary measures are incorporated into concepts of health according to the individual's immediate health concerns. These preventive behaviors are performed to avoid the risk of ill health and in order to preserve and maintain good health. Oftentimes, these measures were encouraged or heightened by the diagnosis of illness, weight gain, surgery, or some other cause for alarm in oneself or in someone close to the individual. Alerts to the state of health often come in the face of problems. 
Alerts were varied: interviewees spoke of clothes not fitting, noticeable weight gain, illnesses, or diagnoses in self or others (though, interestingly, others were only mentioned in the face of illness or diagnoses). Shortcomings in the project of health or the notion that not enough is being done in terms of achieving health resulted in an individual's increased strategizing and surveillance of behaviors and choices in an attempt to ward off future problems. As such, the specific precautionary measures that an individual takes are incorporated as part of an individual's health concept:

“...my mom had cancer and so that, breast cancer, she's all right now, but that makes you think that, you know, you need to start watching what you eat... and my grandpa died a couple years ago and he had heart surgery and it was coming, I mean, he was diabetic, so that, you know, when you have so many family members who are around you, you really - if not for physical appearance, just for the simple fact to be healthy, to not have medical problems when you're older, not as many" $(\mathrm{C} 10$; age 20).

"I have a pretty good family history of heart disease and um, even some cancer and so, yeah, that's a big - yeah, especially as I get older, it weighs a little heavier on my mind. I'd say it's daily because my diet is part of it, so yeah, that's something I'm conscious of" (C15; age 25).

Self-surveillance and self-responsibility

The achievement of health is considered to be contingent upon personal effort and responsibility toward this end (Armstrong, 1995; Frank 1995). Disciplinary tactics such as self-surveillance, self-responsibility and self-monitoring were expressed in the interviews. Study participants discussed actively taking care of their health through self- 
surveillance techniques. Participants identified the monitoring of her behavior as key to keeping herself healthy and in top condition:

(What immediately comes to mind when you hear the word "health"?) "I think of taking care of yourself and the things that you can do to take care of yourself - like, actively" (C17; age 24).

(Who exemplifies health in your opinion?) "Venus Williams or Serena Williams...because I'm sure that they have to surveil themselves in everything that they eat, I'm sure they have dieticians that they interact with daily and they're so physically fit, you know, they're very body conscious" (C21; age 23).

When interviewees discussed their healthy lifestyle practices, they alluded to the responsibility they felt for their own health. This responsibility was activated through dietary regimens, exercise routines, stress reduction techniques, and so on. One participant was very clear in stating that the locus of responsibility is her. She critiques the idea that you have "no control" over your health and firmly states that people are in fact in control of their health:

"I was depressed, I wasn't happy and so that was really a point where, you know, I had to come to terms with...the responsibility that I had over myself. And that's really - that's...the responsibility that you have over yourself is the responsibility that you have over your own health and you know, as much as you can, to take care of yourself...in terms of what you can do to take care of yourself and to keep yourself in as good of shape as you can be in, you know barring all other diseases... I reexamined that and I reexamined my own -I guess the control I have over my health. You know, and, just because it's easy to assume 
that you have no control, but I think that you have a good deal of control over the shape that you're in on a daily basis" (C17; age 24).

The notion of self-responsibility for health was expressed by my sample participants as the motivation to participate in certain health behaviors. For example, interviewees expressed an attentiveness regarding healthy nutritional intake and participation in physical activity. If she expressed the desire to restructure her diet, for example, by choosing to eat more fruits and vegetables or avoiding red meats or processed foods, then she also expressed the awareness that responsibility rests upon her shoulders to eat in this manner. Considering health as a contingent characteristic, the individual learns that her health is somewhat dependent upon the behaviors that she devotes toward her pursuit of health:

"The days it's nice, I walk on my lunch hour. I try to go to the gym a couple of days a week and workout and some weeks it works and sometimes it doesn't fit. So, I mean, I'm conscious of that and I do feel guilty when it doesn't go the way...you know, when I can't fit it all in...Like, I should have exercised and I know that I need to do this" (C19; age 55).

"I pay attention to my diet, my eating habits; I've done a lot of restructuring over the past week as part of the yoga class and as part of my own initiative to kind of pay attention to salt and fat intake and modify those..." (C15; age 25).

Self-surveillance allows the individual to monitor these health-related behaviors, and to track progress or defeat. Self-surveillance requires the acceptance of responsibility 
for health. Interviewees expressed the responsibility they felt for making "healthy" decisions or replacing unhealthy behaviors with healthy ones. Using olive oil in cooking and prioritizing time for an evening walk over time for an evening in front of the television are choices that interviewees made, thereby increasing her feelings of health.

Study participants discussed surveillance as a way to avoid weight gain, prevent diseases and track the amount of daily health behaviors:

"I've gained some weight and not that I feel like - I mean I don't have an issue like "Oh, I'm fat," but it's more like I know that I'm getting to the age where my metabolism is going to start slowing down and I need to be aware of eating portions that are not just eating because I'm bored....and I know that I, when I get tired at night, I tend to want to eat and just being aware of that and my eating habits and eating in a way that gives me what I need and not just because it's fun to it. I love to eat so my weight has been a bit of a concern - not like a major concern but it's like, I don't want it to keep creeping up and creeping up because I' $m$ eating the way I ate when I was 23 and now I'm almost 30 or whatever... Um, so that would be an issue" ( $\mathrm{C} 9$; age 29).

"I used to not pay attention to what - I mean, I don't mean to be redundant about this exercise and food, but - I used to not exercise regularly and paid attention to what I ate but not great attention. And then there was a turning point for me when my mother was diagnosed with breast cancer and I thought, Oh! You know, I should really pay more attention to my lifestyle...especially as I've become more independent, I just thought, I really need to pay more attention to this... You know because I don't want to look back and regret that I didn't exercise more and I think that's something that my mother does. So, for me, I can actively change that and it becomes something that I really enjoy" (C25; age 24). 
Consumer culture products and images

The contemporary consumer oriented American marketplace is saturated with products and materials specifically designed to aide in the pursuit of good health (Bunton, 1995; Phillips, 2005). As citizens of contemporary consumer oriented American society, my study participants may learn that health and fitness are made possible largely through available external materials. In some interviews, the route to achieving "health" was discussed in relation to molding her diet to a healthier cultural ideal, reducing the size of her clothing, or sculpting limbs by gaining muscle definition and tone. The word "health" conjured up various images that suggested consumer culture influences. For example, some of the women in my sample equated the elliptical machines and gyms with people who are healthy and engaging in healthy behaviors. The food pyramid was often cited as a "reliable" source for nutritional guidance. For some participants, "health" conjured up an image of proper diet and exercise, two behaviors which relied on products and images sold by consumer culture.

\footnotetext{
"Well, the food pyramid kind of comes to mind immediately, that's what comes to mind. And just a row of ellipticals with people on them" (C7; age 24).
}
"The gym...a gym full of people, basically, is the image that comes to mind. People trying to get in shape, people paying attention to their lifestyle" (C15; age 25).

Interviewees learned, from various sources, about particular strategies or practices that would produce health. The women often cited reliance upon external guidance for this knowledge; this guidance came from the expertise of doctors, "very fit" or "very 
healthy" family members or friends, yoga instructors, or published news and literature. Interviewees discussed a kind of "folk knowledge" about health practices that, while medicalized and retaining legitimacy attached to medical authority, was not necessarily rooted in a particular informational source. Interviewees privileged the knowledge over the source, which meant that they participated in certain health-related behaviors because they "knew" they were healthy, but did not necessarily remember why.

“...fiber, I try and get my 20 grams in (How'd you decide on 20 grams?) Um, I don't know, that's the recommended amount... (Do you remember where you read it?) I don't know, some nutrition research or something that I came across for some reason...who knows...I always run into so much" (C7; age 24).

“...what I've been really into is the food pyramid and thinking, I mean, balanced diet and how can you go wrong? (Why do you trust that source?) I don't know, I mean, I guess just because it's everywhere - everywhere, everywhere. And on every package of food there, and I don't know, I've never questioned it before - I guess it's just been engrained in me growing up, like you should eat from all of the food groups and this is how many you should have of them" (C3; age 23).

Although individuals did not passively embrace these images, and, in fact, demonstrated a certain amount of resistance which the next section demonstrates, these culturally available messages did play a role in shaping interviewees' health concepts. 
Resistance

Participants did not appear to be uncritically accepting of or passively embracing health messages from government, capitalist, and media sources. The interviewees were active in the process of constructing their notions of health and their participation in health behaviors. They demonstrated resistance to the cultural norm of "fit" and "healthy" as embodied only as a thin, toned and sculpted body. My study participants did not equate being healthy and fit with just being in shape or eating correctly. Health was conceptualized by participants as more of a holistic embodiment.

Several participants were questionable about weight as the only measure of a person's health, which was discussed as a resistance to the cultural norm of "fit" as healthy:

"I realize that just because you are thin or heavy doesn't necessarily equate to healthiness because I know there are a lot of thin people that are really unhealthy, so while that is sort of a concern of mine - or something that I'm always aware of - I don't equate healthiness with weight...solely" (C22; age 36).

“...when I hear the word health, I think of the body and physical health and exercise. (does a healthy person look a certain way?) No, I don't think of any certain type of body - like, you know, I don't think of muscle, a muscle man or Venus or Serena Williams or anything - I just think of somebody who exercises and eats well - and I mean, I guess there is sort of a physical picture because they are not going to be obese if they are doing those things, but...even if they're bigger, I would still consider them healthy - no, I don't think of a certain body type" (C23; age 27). 
Some interviewees were concerned about their outer appearance, which did influence the way in which they visualized health and how they set specific physical goals. For these interviewees, certain surface-level goals served as at least part of the motivation for engaging in some health-related behaviors:

"I also want to look good and feel good about myself, and so that is another reason why health is important. (When you say, "look good"...?) I mean, appearance - staying fit-looking, making sure I don't become overweight. (Do you have an image that pops into your head [when you think of "looking good"]?) Yeah...I think that the first thing that a lot of people think of is like the people that are in those fitness magazines that, you know, look perfect. But I try to not but you can't help it you know, a lot of times, because that's just what ... those are the pictures that go with the machines" (C8; age 19).

"I'd like to go down one dress size and pants size. And yeah, I'd like just to be a little firmer, you know...it's all getting really soft and mushy...and I'd like to be firm. I want to keep doing those weights because sooner or later, you know, those wings on the bottom of my arms will go away!" (C19; age 55).

While individuals were not immune to cultural pressures, they seemed to be very critical of the notion of uncritically accepting cultural ideals that would have them shape their bodies into forms that, according to interviewees, were impossible. Women suggested cultural bodily ideals that they knew existed. For instance, women suggested a preference for a lean body as opposed to an obese or flabby one. Some women pinpointed certain areas that they felt "needed improvement." There was recognition of these cultural ideals, to which interviewees then used as a comparison to their own bodies or 
health behaviors. Yet, there was a certain amount of resistance to these cultural ideals as well. Women recognized cultural ideals but in talking about their personal goals for health, these ideals did not frustrate their feelings of success. This resistance occurred for several reasons: women recognized that a certain body type did not necessarily represent "health"; women recognized that they had a certain body type that could only be molded to a certain extent; and, perhaps most importantly, these women did not express a strong interest in pursuing a particular body type. Instead, women were more interested in finding healthiness and happiness in terms that matched their ability, resources, and interest:

“...but now I'm very aware of my body you know (so how does that change things?) Well, I, you know, I'm not petite first of all - I'm you know, large-chested, that's never going to change...I think that you have to be realistic about how you're going to look, even at what you feel like is your best, or what I feel like is my best, I guess. But I have to be realistic - even at my most healthy, I have a long torso and there's nothing I can do about it. I'm taller, so I have longer legs and longer arms, you know, just kind of that mold that you're going to fit into. I think a lot more in high school, I thought a lot more about this mold thing and that's never going to happen - and so, you just have to be realistic about what you can achieve" (C17; age 24).

(Do you find yourself comparing your body to other women's bodies?) "I used to - I don't anymore. I think I have just come to the level of acceptance that this is what it is and that I cannot um, be, I mean I think I have come to understand the limitations of my body better and kind of try to understand my body better. It goes more towards, having developed more self-esteem and that sort of thing. I have come to a mental state where all of 
those things don't bother me anymore" (C6; age

28).

Physically Fit vs. Healthy

The importance of word connotation was emphasized when the words "exercise" and "gym" were contrasted with "physical exercise." Many interviewees expressed extreme displeasure in the thought of exercising and the idea of making regular trips to the gym, yet would gladly participate in physical activity. Others avoided the use of the term "exercise" altogether, opting instead for terms that they considered to be positive alternatives, such as "activity" or "fitness." Moreover, to describe their own participation in physical exertion, some women were careful to use specific language, due in part to the belief that by using the word "exercise," she was thereby committing to something to which she didn't necessarily feel willing, ready, or desirous to commit. In finding alternatives to the term "exercise," participants consequently expressed a desire for control, choice and agency in adopting health behaviors.

One participant shunned the word exercise, stating that the very word was laden with negative connotations:

"Exercise is, I think, kind of a bad word - I think negatively when I think of exercise, but...I mean, just like if you say "rollerblade," that's exercise but if you call it exercise...(So there's all these connotations with exercise - in order to exercise, you'd have to go to the gym and...) Yeah, and workout and run on the treadmill - I hate running on treadmills - it's just, it's a bad word. (Do you like the word "fitness"?) Fitness. I can deal with fitness. Fitness is more positive and it's staying in shape and endurance, yeah..." (Code 10; age 20). 
Another interviewee contrasted the physical activity and physical fitness:

\begin{abstract}
(What do you think of when you hear the word "fitness" or "exercise"?) "I guess being physically active but making a point to do so, not just the every day walking, climbing steps that you... (walking up the steps is exercise, but you don't see it as physical fitness?) Right. (ok, so physical fitness is more...what?) Physical fitness is more setting aside a time in your day or every few days or whatever to, to only do exercise. (does it have to be at the gym?) No, it could be at the gym, it can be at your home, uh, it can be running around your neighborhood - it can be anywhere - but a time -I guess I always think of it as a time that you specifically set aside for that reason" (C17; age 24).
\end{abstract}

For some women, the word exercise conjured up images of fit bodies participating in high-impact exertion and wearing sweaty spandex at the gym. These women were much more comfortable with a term that, in itself, did not seem to have such stringent requirements. One interviewee commented that the term "activity" was much more flexible in what it required from its user. She purposely avoided using the word exercise to describe her own participation in "physical activity":

“...finding ways to kind of fit in activity - not necessarily exercise - I really do hate exercise, but I mean I do it because I don't want to die at 35 but $u m$, and so I um, and so I do exercise but I'm trying to find ways to sneak kind of fun activities in there. (Like what?) I love to take walks and we live in a great neighborhood that has a lot of stuff to do in it. And, so it's nice being able to kind of walk and say, "Ok, today I'm just gonna walk while I do my grocery shopping," or, "Today, I'm going to walk while I drink my coffee or whatever" (So you see that kind of thing as exercise?) I see it more as activity. Exercise to me is defined as, you know, when your heart rate is supposed to hit that certain 
point and blah blah blah... When I say "your heart rate hits a certain point," like you're supposed to be exercising at you know at, what, $80 \%$, or something like that, to really burn fat or whatever. And so, to me, that's really exercise. The walking, I can kind of go at my own pace, I don't have to go for, you know, a pretty hard pace for 45 minutes - I can just go and have activity" (C18; age 26).

At times, exercise was spoken of in terms of being "too much" or "too strenuous" - even unhealthy - for an individual. As a result, individuals opted for activity that was easier on the joints, more appropriate for certain body types, or more adaptable to everyday life:

"But um, as far as fitness, the biggest thing right now is the "rebounding" and I've been doing that since last fall (and that's just...) Yeah, it's just a little mini-trampoline, about 3-feet in diameter, and I do that about 10-12 minutes each day...it's really a lot of fun. And it's not, you know, it's low-impact so it's really good for the joints. I'm not a runner, you know, I'm big-chested and there's not enough bras... and I've got, you know arthritis runs in my family, so you know, and I just don't enjoy running. You know, I love walking... The walking is lowimpact, but the rebounding is low-impact. (So you've always been pretty active?) Oh yeah, yeah - even if I didn't have a regular exercise routine, you know, prior to my previous dog, I was very, very active. I love working in the yard, cleaning... (Ok, so you see all of that as activity and exercise?) Yes, yes - you know, going up and down the stairs, I'm very, very, very active" (C20; age 37).

"I try to incorporate exercise into my day somehow and typically that includes walking my dogs - but it's not, I mean I don't really get a whole lot of exercise really, it's sort of low-impact exercise, about 20 - it takes me about 20 minutes to walk 
them - and then walking around campus is included in my exercise, sort of - not regimen - but, habits...(so you really enjoy walking) Yeah, I do. I mean, I think running is more profitable in terms of quicker transformation of your body, but I just don't prefer to run..." (C21; age 23).

Alternately, though adding to the notion that the gym offered "exercise" while outdoor or daily activity offered "fitness" or "physical activity" was the notion that in order to be "exercise," she had to be at the gym or in a class, and in the case, there was a preference for such:

(Does the gym feel more like exercise than the walking?) "Definitely (Is it because you're working harder, or because of the environment, or...?) No, I think it's because I'm working harder, you know, I've broken into a sweat, I really feel good... I mean, I really do think that when I'm at the gym is like my "for real" stuff and the other stuff is I guess, like to maintain" (C12; age 24).

"I think when I think about fitness, I mostly think of cardio activities and not so much things like yoga. (gardening?) Yeah, that can be ... like for my mom or my dad" (C5; age 24).

Other interviewees expressed discomfort with going to the gym, as they believed themselves to be unfit for an appearance there. This discomfort illustrates how societal expectations influence some individual's concept of what "fit" bodies look like:

"No it doesn't really appeal to me. I guess also, I mean, the gym, it just seems like a little more...I can't think of the word to describe this, but they want you to dress a way, it's almost a social thing to be at the gym, too. It seems like people always want to go in groups or something. And, when I did go to 
the gym, I went with friends, but I don't know, I just get bored (So, you said, you feel like you have to dress a certain way?) I guess I don't really feel like I fit the gym rat profile. (And what would that be?) I think of preppy, athletic guys, actually. And I just don't want to work out with them. Not, that I don't have preppy, athletic friends, but I just ... I don't feel comfortable I guess. (When you go to the gym, do you feel like you have to wear certain clothes?) Yeah, like when I go to yoga class, sometimes I just wear my pajamas because it's all about being comfortable and that really appeals to me. Yoga seems, it's more of an internal and external exercise too...yeah, it reflects my own philosophy" (C1; age 21).

(Do you go to the gym at all?) "No, I go in there and get, you know, intimidated by all of the already-fit people and I just kinda go, ok we're going to go home...I just feel like I don't exactly what I ought to be doing and therefore, all of these other people in there that do know - obviously, since they're already fit - would be like, what's this moron doing? So, I just, I don't want to embarrass myself, so I just go home" (C16; age 20).

Many women spoke of their preference for outdoor activity that was neither too strenuous nor too high-impact. Women mentioned walking, yoga, biking, and swimming more so than running, although two of the women were currently training to participate in a mini-marathon. Yoga and pilates were the fitness preferences most often cited; they were mentioned by eleven participants. Overwhelmingly, the preference was for everyday activities as easily incorporated into an individual's routine. Many women conceptualized exercise as a daunting task, one that required hours of sweating at the gym. Those who conceptualized exercise as a daunting task often preferred to participate 
in physical activity that occurred outdoors and/or was one of the aforementioned lowimpact alternatives to "exercise."

Healthy eating vs. dieting

The concept of healthy eating was separated from the concept of dieting in interviews. Healthy eating was spoken about in terms of balance, moderation and flexibility. Dieting, on the other hand, was spoken of as being too strenuous, rigid and unpleasant. This delineation between healthy eating and dieting illustrates how study participants actively constructed an alternative to cultural ideals related to food consumption. Although cultural images of "proper" eating can be linked to specific mainstream diets (such as Weight Watchers or the South Beach Diet), participants were not accepting of these dietary practices as healthy. Instead, participants expressed a desire to eat healthily with a limited amount of external rules and proscriptions. While healthy eating was a critical component to all participants' concepts of health, dieting was not necessary - and in fact, was often cited as unhealthy.

For many participants, healthy eating involved concepts that they grew up with, that they remembered being a part of childhood meals or lessons.

“...growing up...I always ate, you know, fairly healthy. I mean, we had a balanced dinner every night, which I think is key to being healthy, and you know, your mom packs your lunch or you eat the school lunch which is bound to be healthy and then, breakfast. So, yeah, I feel like, and then I guess when I came away to college, it was kind of like, ok, I'm the one who has to make these decisions, so I guess just now, maybe, am I consciously thinking, ok I need to eat from the four food groups and I need to be active" (C3; age 23). 


\begin{abstract}
(when you say 'eating right' what does that
mean?) "Getting your pyramid of fruits and vegetables and grains and dairy - I mean, my mom raised us to think in terms of that, so I do think, how many fruits have I had today, how many vegetables have I had today and not eating fast food and uh, not eating fried food. I was taught that fried food was evil - or only for very special occasions - and basically, just watching your sugar - always watching your sugar..." (C2; age 30).
\end{abstract}

Eating healthily was a practice which many participants learned from their habitus. Rather than using cultural messages of "proper eating" as a guide for nutritious eating, most participants referred to the ways in which they remembered eating while growing up. Interviewees favored the familiarity in eating habits that they learned within their family over the cultural recognition of mainstream diets:

(Who exemplifies health in your opinion?) "My parents. (Why?) Well, we've always had our own garden. We've always had a big garden...We ate a lot of vegetables, a lot of rice - a lot of vegetables and a lot of rice, actually. I don't know, I mean I guess I didn't even realize it until I did move away on my own and all the food that I was eating, was uh... This isn't really like food...for some reason I'm craving vegetables. This is so weird because I hated them when I lived there. But my parents definitely set the example for me. Just how easy it is to make it a part of your life, really if you just do..." $(\mathrm{C} 1$; age 21$)$.

"I grew up in a household where, it was sort of meat and potatoes - but always vegetables and, you didn't eat dessert until you had vegetables and things like that... we enjoyed salads, fresh vegetables, fresh fruits, um cooked vegetables...And, I grew up learning how to bake and so if we wanted something it was - ok, there's eggs, sugar and flour, go at it. But again, that was 
part of our diet, but not the key component of our diet and it was, you know you eat your meat and vegetables and fruits and things like that and then, you know, dessert is part of it. But, that just was not a huge issue for us. So...yeah I think I've always been healthy. (ok, so you feel like you've always just been healthy - it almost feels normal) Yeah, exactly, exactly. I would be, I would miss vegetables in my diet - and you know, not like French fries. I would miss a salad or you know...so I think I've always felt healthy" (C20; age 37).

Healthy eating was spoken of as a relaxed, "feel good" practice. The characteristics of "relaxed" and "feel good" were referred to as necessary components, in order to remain moderate, balanced and "healthy" when it came to eating:

"Finding something that, that you're fueling your body with the healthiest fuel you can give it and still enjoy eating and see it as an adventure and sort of approach it with a light heart and curiosity and sort of, what can I discover about this, rather than that heavy "I should do this" or 'I shouldn't do that,", (Code 9; age 29).

The act of eating was spoken of in terms of being a fun, social activity. In this case, participants were careful not to let eating rules and regulations overwhelm the social setting. Healthy eating was "healthy" when it did not limit the participant's desire to socialize, have fun, and enjoy herself.

"So much social activity surrounds food, especially social activities involving desserts and sweets and cake and stuff - and you know, you don't want to not be participating" (C17; age 24). 
Dieting, on the other hand, was separated entirely from the concept of "healthy eating." In fact, dieting was not a practice of which interviewees spoke fondly.

Oftentimes, the idea of restricting herself too much or trying to adhere to a severely limited nutritional intake was considered harmful or unhealthy as opposed to beneficial or healthy.

\begin{abstract}
"And some of my friends try these crazy diets, like all they're going to eat is broccoli (are they girls?) yeah, they're on the crazy diets. I guess that's another reason why I don't want to be obsessed about it because I see people that it really kind of controls a part of their life and I don't think it makes "em happy" ( $\mathrm{Cl}$; age 21$)$.
\end{abstract}

"I would say... a nice little portion of my friends try to eat very healthy and very organic - and, I don't go to that much extent just because, um, because Iyou know, it's like too much for me. Too much, too many limitations (ok, so too much to think about; taking it to a new level?) Yeah! For me, for where I am in terms of food - it almost, like I do, I really enjoy food; I really enjoy cooking and it almost makes it un-fun when you do all that...for me..." (C17; age 24).

\section{Health Identity}

The concept of health identity emerged from the twenty-five documented health accounts. As study participants, self-proclaimed "healthy females," described their concepts of health and health behaviors, elements of both structure and agency emerged. That is, while socio-cultural influences were acknowledged as part of the construction of health concepts and health behaviors, an active resistance of cultural and medical 
messages of health that seemed unattainable or undesirable played an equally significant contribution to the construction of health-related concepts and behaviors.

The participants in my sample spoke about health as a personal, achievementbased entity. Rather than a "state of being," health was discussed by participants as a consciously negotiated achievement. The obtainment of health expresses qualities of the self, both morally and in terms of lifestyle. Health identity is an expression of the habitus, since this form of identity is shaped, in part, by the norms, patterns, and habits of an individual. Health identity involves the reproduction of class, as it is within the cultural field (which is characterized, in part, by class) that health identity is formed, and, therefore, reproduces the class in its formation. The concept of health identity incorporates the self-surveillance, a pressure to which most interviewees alluded. While the degree and focus of this surveillance varied, many interviewees spoke of regulating their eating habits, monitoring their activity level, and improving or increasing their participation in health-related behavior. The concept of health identity also incorporates the idea of resistance; here, this resistance was against cultural norms and ideals for body image and healthy behaviors. As my participants spoke about the construction of their health concepts and health behaviors, they spoke clearly about resistance to ideals (such as a "flawless diet" that included no fats or sweets or an extremely thin, supermodel physique) that seemed unattainable and "unhealthy." Thus, there was recognition of as well as resistance to cultural ideals. This constant negotiation process that each participant experienced, and reflected upon in her interview, led to the concept of health identity. 
The construction of health identity involves government and capitalist health messages as well as a personal resistance to these messages. Foucault's notion of biopower illustrates governmental interest in healthy, fit and productive bodies for the purpose of increasing benefits for the state. Further, health identity involves an individual's habitus, Bourdieu's contribution that describes how an individual's social situatedness impacts the construction of identity - in this case, health identity. The messages produced by government and capitalist forces were not necessarily adopted absent of critical thinking by study participants. Rather, the notion of health identity develops - and changes - over time and as a result of an individual's social situation: what he or she has come to recognize as "normal" based on familial, gender, economic, education, ethnic, and racial factors. Further, the concept of health identity recognizes the variation in individuals' definitions of health and health behaviors over time, across locations, and from person to person. Health identity captures the construction of an individual's ideas, beliefs and practices as they relate to health. 


\section{DISCUSSION}

Study participants spoke about their concepts of health and health behaviors as constructed via influences from both human agency and larger social structures. There was not a single source that necessarily determined a woman's concept of health; rather, health was discussed as emerging from the influence of a variety of sources: cultural messages, social factors, familial influences, experiences, morality, politics, and so on.

Culturally available messages of health influenced the participants' concepts of health and health behavior. These messages asserted a certain amount of control over the ways in which health was conceptualized (e.g., what a "truly" healthy diet is; what a "fit" body really looks like). There are elements of control that set the parameters of the women's resistance and involvement in health behaviors. Dominant cultural images associated with health were important in how participants spoke about health, but when talking about their behavior, "health" was presented as an alternative choice to consumer, aesthetic body ideals that are expressed as oppressive, unhealthy and unattainable. The women in my sample discussed "health" as having positive moral connotations of being an achievement, which was, in part, expressed as contingent upon an appropriate level of consistent self-surveillance and self-responsibility.

The women referred to cultural standards of health in terms of ideals: the ideal diet, body, exercise routine, and set of health-related behaviors. These ideals served 
primarily as a comparative base rather than a standard to meet or exceed. For example, many women defined the "healthiest" diet as one that was balanced, contained large quantities of vegetables and fruits, was low in saturated fats, and high in fiber, calcium, iron, and other essential vitamins and minerals. The same women who conceptualized this as the perfect diet, however, often admitted to not matching it in their own nutritional intake. Rather than expressing guilt or remorse over this reality, the women conceptualized their own "imperfect" diets - defined as one that did not always have enough daily fruits or vegetables, that included desserts, and that sometimes was just not "up to par" - as part of their health identity. The USDA food pyramid, a government standard of healthy eating, was often cited, for example, as a model for diets. And yet, its guidelines tended to serve more as a suggestion than a rule. Resistance to this cultural expectation for the "perfect" diet (e.g., one that flawlessly adheres to USDA guidelines) was present in nearly every interview.

A similar ideal in terms of a perfectly "fit" body (e.g. thin, toned body parts, sculpted abdominal muscles, etc.) was recognized by interviewees. For these women, however, these ideals were not turned into sources for discontentedness with their own bodies. Rather, the ideals were recognized along with the "reality" that this ideal was not possible for all body types. Many girls discussed a process of getting to know their own bodies and, in turn, learning what expectations (in terms of physical results) were possible and which ones were not. Many girls stated that these standards were simply not feasible and, moreover, not particularly grieved over. The "fit" body type did, at times, serve as a source for avoiding the gym, as the individual did not feel she "belonged" or "fit in" at the gym because of the multitude of fit bodies. Other individuals avoided 
committing to excessive exercise regimens, as this seemed to bring their participation in fitness to a new, obsessive level with which they were not comfortable. These cultural messages of "fitness" were recognized by my participants, yet they were not uncritically incorporated into their health concepts and health behaviors.

The participants expressed resistance to dominant culture bodily and aesthetic ideals. This is a resistance, however, that is defined in dominant cultural terms. Foucault's concept of bio-power should be revisited in order to understand how participants' concepts of health and resistance to dominant ideals of health are affected by bio-power. Bio-power, in its dispersion throughout society and as enacted through bodies, analyzes, regulates, controls, explains, and defines the human subject, its body and behavior. The concepts of health, participation in health behaviors, and resistance to unattainable cultural bodily and aesthetic ideals are all negotiated within a hegemonic health discourse. The effects of bio-power in constructing health identity are critical to this analysis and an understanding of the significant role that governmental power over life, or bio-power, has on the construction of health identities. Among the effects of the dispersion of bio-power is resistance to this type of power, which was also present in these documented health accounts. Study participants expressed resistance to cultural messages of health when these messages seemed unattainable or unhealthy, or when the messages were not congruent with the participant's habitus.

The health concepts and health behaviors represented in this study reflect the habitus of primarily White, mid-Western, middle-class females. As such, the "image" of health and the engagement in health behaviors reflect the norms most closely associated with White, mid-Western, middle-class females. For example, the unattainable cultural 
body ideal most prevalently resisted was that of the super-thin, nearly-anorexic supermodel. This image is typically portrayed as the ideal for females of White, midWestern, middle-class status. Similarly, many participants cited the USDA food pyramid as a guide for nutritional eating. While this nutritional guide is displayed on certain packages of foodstuffs (e.g., frozen vegetables, whole wheat bread, rice), it is not typically displayed on others (e.g., bags of potato chips, cookies, packaged meats). Depending upon the foodstuffs that an individual purchases, then, he or she will be variously exposed to this resource and variously exposed to the foods (and specific ratio of these foods) that it purports as "healthy."

Because the concept of health identity primarily reflects the habitus of White, middle-class, mid-Western women, this concept reflects lifestyle choices (diet, exercise, weight control) as the road to good health. As such, social conditions (knowledge, money, power, social connections, access to health care) are not necessarily the first and foremost considerations for my study participants, despite their overwhelming influence on an individual's health (Jimenez, 1997). The specific health identity of my sample health as a project involving self-responsibility, self-surveillance and personal, daily achievement - may not be an accurate depiction of health for an individual who does not have regular access to health care and whose health is particularly affected by other social variables. Nevertheless, the concept allows for an understanding of health as a fluctuating concept, one that is tied to an individual's social situatedness.

The influence of popular culture played an important role in my study participants' "image" of health, which appears to derive from popular culture, such as a diet that includes "healthy" foods and participation in regular exercise. However, in 
constructing their own health identity, these women transform these images into concrete practices that, while quite different from the popular culture "image," allow them to feel "healthy." Health identity allows an empowering construction of health as an alternative to the cultural dominance of media and consumer body ideals, and in this way, health is an alternative positive embodied identity. 


\section{LIMITS OF GENERALIZABILITY \& CALL FOR FUTURE RESEARCH}

I developed the notion of health identity from the study findings presented above. Most participants were either attending college or graduate school or working within the university, which also influenced the pool from which participants were recruited. Most participants were white and grew up in middle-class households, which limits the cultural fields that were represented. The concept of health identity, which currently reflects these particular ethnic and socioeconomic variables, calls out for replication in different sociological groups. With more class-based diversity, the data would reflect norms of individuals from different cultural fields and different conceptualizations of normalcy. Due to the limitations of this study, the findings are not generalizable, though generalizability was not a goal of the study. Instead, the findings, which do call for comparative analyses, describe only this specific population of women. Despite the limited ethnic and socioeconomic diversity among the participants, as a preliminary study of health accounts, this study may pave the way for additional studies of its type, some of which incorporate diversity into the criteria for recruitment since situatedness and habitus are key concepts that must be examined via diverse populations.

Health identity is an expression of an individual's habitus and is therefore situated within a particular social class. The concept of health identity should be translated as social structure / human agency, but the specific content varies. As such, the concept may 
be specific to the class and cultural field of my sample, which may consequently weaken the robustness of this concept. For instance, the habitus of the majority of my sample participants involved knowledge about health and health practices. Many grew up with parents who prepared healthy meals and many participated in sports or engaged in physical activity with their families. Furthermore, most of the women have structural access to healthy living (e.g., gym memberships through college enrollment, a nearby park, a bike, or enough money to purchase healthier foods or books about healthy behavioral practices). Because I constructed the notion of health identity based on the accounts of health concepts and health behaviors of a narrow sample of women, more research should be conducted to assess whether the concept of health identity extends as well to other samples. For instance, the concept of health identity may not be relevant to poor, Latinas living in America who cannot speak English, as the habitus of these women may not express similar knowledges of health, which influences the type of health identity that emerges. While the robustness of the concept of health identity should be tested, this concept does predict different interpretations of "health."

The current study offers many opportunities for future research. The scope of the study did not allow all possible areas to be investigated; indeed, the area of concentration will benefit from additional, extensive study. To begin, the convenient sample was drawn via an advertisement for "healthy females." While this was purposeful, the specific audience that was recruited may live significantly different lifestyles and have significantly different conceptualizations of health than an audience drawn by an advertisement for "physically fit females" or "nutritionally-conscientious females." How would these females' conceptualizations of health and healthy lifestyles differ from the 
ones documented from women who define themselves as healthy? That is, would women who define themselves as "physically fit" incorporate the notion of "balance" within their definitions of health? Or, how would "nutritionally-conscientious" females incorporate fitness into their health practices?

The issue of the language used in recruitment is useful to consider against results from other similar studies. For example, "the desire for 'good health' becomes a very minor component of people's reasons for engaging in exercise regimens, superseded by concerns engendered by the powerful ideologies of morality, asceticism, self-discipline and control which underlie consumption patterns in a culture which is intent upon selfpromotion and achieving 'the look"' (Wernick, 1991; Featherstone, 1991 as cited in Lupton, 1995, 146). These results were not necessarily duplicated in the current study; participants did not seem to steer too far from the desire for "good health." This begs the question of sampling strategies: that is, what criteria were used for participants in the aforementioned studies? The current study required that participants define themselves as healthy; did this impact their embodiment of health to the point that they would not be as concerned with consumption patterns as participants who were not required to view themselves as healthy?

Bourdieu's concept of habitus has been used in some research within the health field (e.g. Singh-Manoux \& Marmot, 2005; Meinert, 2004; Inglis, 1997; Ohman et al, 1999), it has received criticism in others (Crossley \& Crossley, 2001; McNay, 1999). In particular, habitus is criticized as potentially insufficient in explaining the variation in an individual's perceptions over time; that is, the question of change may not be sufficiently answered; "Why and how have the habitual structures which shape the modern user voice 
emerged? How can we explain the appearance of the new typifications and narrative and rhetorical strategies...?" (Crossley \& Crossley, 2001, 1478). Thus, while the theoretical framework was not limited to, nor necessarily limited by, Bourdieu's concept of habitus, future research may benefit from either usefully modifying the current theoretical framework or positioning itself within a different one.

Along the lines of transforming the conceptualization of health is the idea of age differences in health accounts. A comparison between young and older age groups would serve as a significant contribution to research. So too would the exploration of the health accounts of men, as this was left untouched. How do men conceptualize health, to begin; and, of particular interest, how are their dimensionalized definitions different from - or similar to - women's; are there more similarities between men and women of the same age?

Additional insight may emerge from the examination of short-term health fixes such as fad diets or over-the-counter medication - and reliance upon corrective medical services rather than preventative health behaviors. That is, do individuals participate in short-term fixes for our health? Have we been "trained" to do things that help us only in the short term and may not have any effect or benefit (may even create problems) in the long run? While fad diets were frowned upon within the current health accounts, do short-term fixes, such as fad diets, occur within certain groups - such as those who focus more on idealized body images (i.e. the slim body)? What role do corrective medical services, such as cholesterol and blood pressure medications, play in an individual's health? Do individuals rely more on corrective services rather than preventative behaviors? 
Also, the notion of expertise was not explored within the current study. While there was mention of preferences for advice coming from "experts" - doctors, Olympic athletes, nutritionists, friends who recently lost weight - there was no extensive questioning regarding the criteria for "expert" in lay accounts. How do lay persons conceptualize professionalism within the health field? Who do they consider to be professionals (chiropractors? massage therapists? yoga instructors) and why?

The interview schedule attempted to pull out the multi-dimensional definitions that participants have of "health." To do so, the schedule focused on general health, fitness, and nutrition. It may be a valuable exercise to focus just on the section concerning overall health, as this section seemed to invite the most in-depth answers. It may also be valuable with future projects of this kind, to conclude with a focus group including all interview participants, as many alluded to their interest in other participants' answers, as well as the study's findings. Another telling exercise may be to ask participants to read other individuals' "health biographies" and decide whether to accept or reject them from the "healthy group" based on their own definitions of health.

Additional studies which assess the presence of "health identity" in numerous, diverse population samples would also contribute to the robustness of this concept. How does this concept differ across samples? That is, how do health identities of men differ from women, across ages, economic statuses, religious affiliations? And, how do the influences in the construction of this concept differ? Are middle-class, African American men more influenced by the media in constructing their health concepts and healthrelated behaviors than upper-class Asian American women? Are certain population samples more or less resistant to cultural ideals than others? Clearly, there are many 
opportunities for additional research, all of which would provide important contributions to the field of sociology of health and illness as well as the development of health interventions. 


\section{CONCLUSION}

The multi-dimensional definitions of "health" documented and analyzed in this study were definitions that came from women who define themselves as "healthy." Participants were asked to discuss the role of health in their lives in terms of the components and dimensions they see as necessary to be healthy, how their lifestyles and decisions reflect their notions of health, influential people and literature in their project of health, and how, for many, daily consciousness in necessary in this project. The findings suggest that individuals play an active role in constructing their own concepts of health and health-related behaviors. Culturally available messages of health concepts and health behaviors were translated by the individual based on her habitus, cultural field, preferences, and resources. The result was the construction of her health identity. In this way, women negotiated health concepts and behaviors in an active, empowering way. The interviewees were not passively embracing the messages and images being sold by government, capitalist, and media sources. Rather, the women resisted, to a certain degree, the images and patterns that are set forth by these cultural and social sources. The resistance to these messages that each individual had was shaped by her habitus and cultural field. The women are influenced by both government and capitalist messages and a personal resistance to these messages.

In exploring health accounts from self-identified "healthy" women, we find that the culturally available concepts are rarely left intact. Rather, as these health concepts are 
incorporated into her ideas about health, they are negotiated and tweaked so as to fit into her lifestyle in a more feasible way. Rather than following a rigid dietary regimen, for instance, a healthy individual is likely to abide by cultural definitions of a healthy diet but in a loose, more emancipatory manner. As these changes in health concepts occur, the individual begins the process of constructing his or her health identity.

The data from this study offer examples of the negotiation process between wider cultural health concepts and the dimensions of an individual's health identity. This negotiation process refers to the transformation of cultural ideals into a more individualistic project - one that reflects the individual's lifestyle and preferences; one that makes the individual happy; and one that is manageable and feasible in a world of multiple responsibilities. Thus, while the construction of health identity draws from cultural discourses, it is shaped in large part by the active, resisting individual. These findings may be useful in the development of health-related messages and curriculum produced by health intervention programs, health promotion campaigns, health educators, and healthcare providers. As the quest for health continues to grow in America, a greater awareness of how health identities are constructed may pave the way for more effective health-related messages and intervention programs. Health identity is constructed within a hegemonic health discourse, yet it involves a negotiation process so that both individual agency and social structures play a role in shaping how health is conceptualized and how the individual participates in health-related behaviors. 


\section{REFERENCES}

Albrecht, G. L. (1999). The marketing of rehabilitation goods and services. In K. Charmaz \& D. A. Paterniti (Eds.) Health, Illness, and Healing: Society, Social Context, and Self. Los Angeles: Roxbury.

Armstrong, D. (1995). The rise of surveillance medicine. Sociology of Health and Illness, 393-404.

Barrett, B. (1995). Ethnomedical interactions: Health and identity on Nicaragua's Atlantic coast. Social Science Medicine, 1611-1621.

Baudrillard, J. (1998). The Consumer Society: Myths \& Structures. (Translated by Chris Turner). California: Sage.

Bolam, B., Murphy, S., \& Gleeson, K. (2004). Individualisation and inequalities in health: A qualitative study of class identity and health. Social Science \& Medicine, 1355-1365.

Bordo, S. (1993). Unbearable Weight. Berkeley: University of California Press.

Bourdieu, P. (1979). Algeria 1960: The Disenchantment of the World, the Sense of Honour, The Kabyle House or the World Reversed, trans. Nice, R. Cambridge: Cambridge University Press.

---- 1986. The forms of capital. In J. G. Richardson (Ed.), Handbook of theory and research for the sociology of education (pp. 241-258). New York: Greenwood.

---- 1990. The Logic of Practice. Cambridge: Polity.

Brown, P. \& Zavestoski, S. (2004). Social movements in health: An introduction. Sociology of Health \& Illness, 679-694.

Bunton, R. (1995). Health off the shelf? In R. Bunton, S. Nettleton, \& R. Burrows (Eds.), The Sociology of Health Promotion: Critical Analyses of Consumption, Lifestyle $\&$ Risk. London: Routledge. 
Carpiano, R. M. (2006). Toward a neighborhood resource-based theory of social capital for health: Can Bourdieu and sociology help? Social Science \& Medicine, 165175.

Charmaz, K. (1999). 'Discoveries' of self in illness. In K. Charmaz \& D. A. Paterniti (Eds.) Health, Illness, and Healing: Society, Social Context, and Self. Los Angeles: Roxbury.

Collins, P. H. (2000). Black Feminist Thought: Knowledge, Consciousness, and the Politics of Empowerment. New York: Routledge.

Conrad, P. (1999). Wellness in the work place: Potentials and pitfalls of work-site health promotion. In K. Charmaz \& D. A. Paterniti (Eds.) Health, Illness, and Healing: Society, Social Context, and Self. Los Angeles: Roxbury.

Conrad, P., \& Schneider, J. (1992). Deviance and Medicalization: From Badness to Sickness. Temple: Temple University Press.

Crawford, R. (1979). Individual responsibility and health politics in the 1970s. In S. Reveroy \& D. Rosner (Eds.) Health Care in America. Philadelphia: Temple University Press.

Crossley, M. L., \& Crossley, N. (2001). 'Patient' voices, social movements and the habitus: How psychiatric survivors 'speak out'. Social Science \& Medicine, 14771489.

Danaher, G., Schirato, T., \& Webb, J. (2000). Understanding Foucault. London: Sage.

Doel, M.A., \& Segrott, J. (2003). Self, health, and gender: Complementary and alternative medicine in the British mass media. Gender, Place and Culture, 131144.

Dreyfus, H. L., \& Rabinow, P. (1983). Michel Foucault: Beyond Structuralism and Hermeneutics. Chicago: The University of Chicago Press.

Engs, R. C. (2001). Clean Living Movements: American Cycles of Health Reform. Connecticut: Praeger.

Featherstone, M. (1982). The body in consumer culture. Theory, Culture \& Society, 1833.

Foucault, M. (1975). The Birth of the Clinic. New York: Vintage Books.

---- 1980. Power/Knowledge: Selected Interviews and Other Writings (19721977): Michel Foucault. Gordon, C. (Ed.). New York: Pantheon Books. 
---- 1984. Foucault Reader. Rabinow, P. (Ed.). New York: Pantheon Books.

--- 1990. The History of Sexuality, translated by Robert Hurley. New York: Vintage Press.

Frank, A. (1995). The Wounded Storyteller. Chicago: The University of Chicago Press.

Freund, P. E. S., \& McGuire, M. B. (1991). Health, Illness, and the Social Body. New Jersey: Prentice Hall.

Gauntlett, D. (2006). Theodor Adorno. Retrieved May 5, 2006, from http://www.theory.org.uk/ctr-ador.htm

Gerhardt, U. (1989). Ideas about Illness. New York: New York University Press.

Glaser, B. G., \& Strauss, A. L. (1967). The Discovery of Grounded Theory: Strategies for Qualitative Research. New York: Aldine.

Goldstein, M. S. (1999). The origins of the health movement. In K. Charmaz \& D. A. Paterniti (Eds.) Health, Illness, and Healing: Society, Social Context, and Self. Los Angeles: Roxbury.

Gray, S. (2006, May 4). Organic Food Goes Mass Market. The Wall Street Journal, D1.

Gross, S. J. (1980). The holistic health movement. The Personnel and Guidance Journal, 96-100.

Grzywacz, J. G., \& Fuqua, J. (2000). The social ecology of health: Leverage points and linkages. Behavioral Medicine, 101-115.

Hausman, B. L. ( Notes on understanding Foucault. Retrieved February 25, 2006, from Virginia Tech University Web site: http://www.english.vt.edu/ hausman/ pastcourses/foucaultnotes.html

Hodgins, M., Millar, M., \& Barry, M. M. (2005). “...it's all the same no matter how much fruit or vegetables or fresh air we get": Traveller women's perceptions of illness causation and health inequalities. Social Science \& Medicine, 1-13.

Howson, A. (1999). Cervical screening, compliance and moral obligation. Sociology of Health and Illness, 401-425.

Inglis, T. (1997). Foucault, Bourdieu and the field of Irish sexuality. Irish Journal of Sociology, 5-28. 
Iphofen, R. (2001). Strong words, strong minds, strong bodies: An analysis of the narrative structure of affirmatory metaphors in personal development programmes. In Reframing the Body. Watson, N., \& Cunningham-Burley, S. (Eds.). United Kingdom: Palgrave Publishers Ltd.

Jimenez, M. A. (1997). Concepts of health and national health care policy: A view from American history, Social Service Review, 34-50.

Jones, Y. J. \& Estes, C. L. (1997). Older women: Income, retirement, and health. In Women's health: Complexities and Difficulties. Ruzek, S. B., Olesen, V. L., \& Clarke, A. E. (Eds.). Columbus: Ohio State University Press.

Karp, D. A. (1999). Illness and identity. In K. Charmaz \& D. A. Paterniti (Eds.) Health, Illness, and Healing: Society, Social Context, and Self. Los Angeles: Roxbury.

Kerr, A. \& Shakespeare, T. (2002). Genetic Politics: From Eugenics to Genome. England: New Clarion Press.

Leavitt, J. W. (1999). The growth of medical authority: Technology and morals in turnof-the-century obstetrics. In J. W. Leavitt (Ed.) Women and Health in America ( $2^{\text {nd }}$ ed). Wisconsin: The University of Wisconsin Press.

Levin, B. W., \& Browner, C. H. (2005). The social production of health: Critical contributions from evolutionary, biological, and cultural anthropology. Social Science \& Medicine, 745-750.

Lillie-Blanton, M., Martinez, R. M., Taylor, A. K., Robinson, \& B. G. (1999). Latina and African American women: Continuing disparities in health. In K. Charmaz \& D.

A. Paterniti (Eds.) Health, Illness, and Healing: Society, Social Context, and Self. Los Angeles: Roxbury.

Litva, A. \& Eyles, J. (1994). Health or healthy: Why people are not sick in a Southern Ontarian town. Social Science \& Medicine, 1083-1091.

Lorber, J. (1997). Gender and the Social Construction of Illness. California: Sage.

Lowe, M. A. (1999). From robust appetites to calorie counting: The emergence of dieting among Smith College students in the 1920s. In J. W. Leavitt (Ed.) Women and Health in America $\left(2^{\text {nd }}\right.$ ed). Wisconsin: The University of Wisconsin Press.

Lupton, D. (1995). The Imperative of Health: Public Health and the Regulated Body. California: Sage.

Lynam, M. J. (2005). Health as a socially mediated process: Theoretical and practice imperative emerging from research on health inequalities. Advances in Nursing Science, 25-37. 
Mairs, N. (1999). The desert. In K. Charmaz \& D. A. Paterniti (Eds.) Health, Illness, and Healing: Society, Social Context, and Self. Los Angeles: Roxbury.

Manderbacka, K. (1998). Examining what self-rated health question is understood to mean by respondents. Scandinavian Journal of Public Health, 145-153.

McNay, L. (1999). Gender, habitus and the field: Pierre Bourdieu and the limits of reflexivity. Theory, Culture \& Society, 95-117.

Mechanic, D. (1999). Conceptions of health. In K. Charmaz \& D. A. Paterniti (Eds.) Health, Illness, and Healing: Society, Social Context, and Self. Los Angeles: Roxbury.

Mees, P. D. (2005). Yoga Participation Surges. Physician \& Sportsmedicine, 12-15.

Miller, W. C. (1999). Fitness and fatness in relation to health: Implications for a paradigm shift. Journal of Social Issues, 207-219.

Morantz, R. M. (1984). Making women modern: Middle-class women and health reform in nineteenth-century America. In Women and Health in America. Leavitt, J. W. (Ed.). Wisconsin: The University of Wisconsin Press.

Ohman, A., Hagg, K., \& Dahlgren, L. (1999). Competent women and competing professions: Physiotherapy educators' perceptions of the field. Advances in Physiotherapy, 59-72.

Phillips, B. J. (2005). Working out: Consumers and the culture of exercise. Journal of Popular Culture, 525-551.

Pryce, A. (2000). Frequent observation: Sexualities, self-surveillance, confession and the construction of the active patient. Nursing Inquiry, 103-111.

Radley, A. \& Billig, M. (1999). Accounts of health and illness: Dilemmas and representations. In K. Charmaz \& D. A. Paterniti (Eds.) Health, Illness, and Healing: Society, Social Context, and Self. Los Angeles: Roxbury.

Ratcliff, K. S. (2002). Women and Health: Power, Technology, Inequality, and Conflict in a Gendered World. Boston: Allyn and Bacon.

Sandstrom, K. L. (1999). Confronting deadly disease: The drama of identity construction among gay men with AIDS. In K. Charmaz \& D. A. Paterniti (Eds.) Health, Illness, and Healing: Society, Social Context, and Self. Los Angeles: Roxbury.

Shilling, C. (2005). The Body in Culture, Technology and Society. London: Sage. 
Smart, B. (2000). Postmodern social theory. In The Blackwell Companion to Social Theory. Turner, B. S. (Ed.). Massachusetts: Blackwell Publishers Inc.

Starr, P. (1982). The Social Transformation of American Medicine. New York: Basic Books.

Strauss, A., \& Corbin, J. (1994) Grounded theory methodology: An overview. In N. K. Denzin \& Y. S. Lincoln (Eds.) Handbook of Qualitative Research. London: Sage.

Street, A. F. (2004). Ask your doctor: The construction of smoking in advertising posters produced in 1946 and 2004. Nursing Inquiry, 226-237.

Turner, B. S. (1984). The Body \& Society. London: Sage.

Webb, J., Schirato, T., \& Danaher G. (2002). Understanding Bourdieu. London: Sage.

Weitz, R. (1999). Becoming a person with HIV disease. In K. Charmaz \& D. A. Paterniti (Eds.) Health, Illness, and Healing: Society, Social Context, and Self. Los Angeles: Roxbury.

Wiegers, Y. (1998). Male bodybuilding: The social construction of a masculine identity. Journal of Popular Culture, 147-161.

Williams, S. J. (2003). Medicine and the Body. London: Sage.

Wuest, D. A., \& Bucher, C. A. (2006). Foundations of Physical Education, Exercise Science, and Sport. Boston: McGraw Hill. 
APPENDIX A

\section{Participate in a Research Study!!}

\section{ARE YOU A HEALTHY FEMALE?}

(e.g. exercise, healthy diet, meditation)
All females (18+) who believe themselves to be healthy are invited to participate in an interview-based research study.

Interviews will last roughly 30-45 minutes and will take place ON CAMPUS (Lutz Hall or Malcolm B. Chancey Alumni Center) AT YOUR CONVENIENCE!

Your participation will help advance academic understanding of health in our daily lives.

If you are interested or would like more information, contact the researcher, VALERIE FRENCH, at:

\section{vfrench@gmail.com}

You will receive compensation for your time and inconvenience.

Research affiliated with the

University of Louisville Department of Sociology

Lutz Hall

Louisville, KY 40292

Department Phone: 852-6836 


\section{APPENDIX B}

\section{Dimensions of Health Interview Schedule}

Time Started:

Time Completed:

\section{Introduction.}

Thank you, again, for agreeing to be interviewed as a part of this study.

Before we begin, I would like to cover a few points with you.

\section{Informed Consent}

I would like to go over the consent form with you before we begin.

Do you have any questions about what is on the form, about the study, or about the interview process?

Everything you say to me will be kept confidential to the extent provided by law within the research project. I will not share information with any third parties. When the study is written up, the identities of all the people I have spoken with will be protected.

If at any time you prefer not to answer a question, please let me know and we will skip to the next one. You may withdraw from the study at any time without penalties. Also, please feel free at any time in our conversation to add information you think is important for me to know.

\section{Permission to Audiotape}

The purpose of tape recording is so that I can do a better job of listening to you and not have to write down everything you say while you are saying it. I also want to make sure that I am accurate in my understanding of your responses. May I tape-record our interview?

If yes, turn on the tape recorder and state:

I have turned on the tape recorder. From now on, our conversation will be taped unless you ask me to turn the tape off. Is this okay?

\section{Brief Description of the Study}

The purpose of the interviews I am conducting is to better understand how women think about health and healthy lifestyles. I will not only be using this information in my Masters thesis, but also hope that my research will aid in future developments of health promotion and health intervention programs. 
The interview will last approximately 30 to 45 minutes, but may run a little longer.

\section{Background Questions.}

I would like to begin with a few background questions.

1. What is your age?

2. What is your marital status?

(never married, currently married, divorced, widowed)

3. Are you currently employed?

Do you work full- or part-time?

What type of work do you do?

4. Are you currently attending college or graduate school?

If so, are you registered as a full- or part-time student?

Do you currently receive help with payment for school tuition, books,

food, living expenses, none of the above, all of the above?

5. Do you have a religious preference?

Do you consider yourself to be a religious person?

How strongly do you identify with your religion, denomination, or church?

How often do you attend religious services?

6. How do you describe your ethnicity?

7. Thinking about your family of origin, what do you estimate your household income to be, to the closest $\$ 10,000$ ?

8. Do you have health insurance?

(If yes): What kind of health insurance do you have?

(E.g. Employer-sponsored in own name, employer-sponsored but in the name of a family member, public insurance, private insurance, not sure)

\section{Health-related Questions.}

I would like to begin by discussing about how you think about health.

1. In order to participate in this study, you had to define yourself as "healthy."

Why do you think you are healthy? How are you healthy?

What does "being healthy" mean to you?

2. How concerned are you with being healthy? How important is it to you?

3. When I say the word "health," what do you think of?

What, in your mind, does the word "health" imply?

(Diet, wellness, regimen, nutrition, lower cholesterol/blood pressure, church/spirituality, etc.)

4. What aspects of your health are you most concerned about maintaining or improving? (Some things might be: body weight, overall fitness, muscle tone, 
cardiovascular health, appearance, energy, lack of disease, not getting colds or other illnesses, feeling good, etc.)

5. How do you live a healthy lifestyle?

What does a 'healthy lifestyle' mean to you? What are the most important elements of a healthy lifestyle?

What are the specific things you do to keep yourself healthy? How often to you do these things?

What are the most important things you "do" to keep yourself healthy?

Do you face any impediments in attempting to engage in the activities you think are important for staying healthy? If so, what are these impediments (e.g. time and money)

What are some things you think you should do but do not do? Why do you think you should do these things? Why don't you do them?

6. Can you think of a time when you were not healthy? If so, what was that like?

How do you know you were unhealthy? Or, why do you think you were unhealthy? What was different (in your life) at that time?

7. Who exemplifies "health" in your opinion? Why?

Would you say that your lifestyle is similar to this person's? If so, how? If not, why not? Or, would you say that you use this person as a model for your own life and healthy lifestyle?

8. When did you begin working toward a healthy lifestyle? Has this always been an interest of yours?

\section{Fitness-related Questions.}

The following questions relate to fitness and exercise.

1. How do you think about fitness and exercise? That is, when I say "exercise" what do you

immediately think of, or how do you define exercise?

2. Describe your own experiences with fitness. Do you engage in fitness regularly?

If so, how and where do you get your exercise?

For example, do you always go to the gym to exercise? Do you ride your bike in the park? Do you horseback ride?

3. Describe your typical exercise session...

(E.g., How often do you exercise? Do you exercise with other people?

Do you have an exercise schedule / regimen? If at the gym, do you do the same exercise (or use the same machine) every time you are there? Do you vary your routine? What do you incorporate in your fitness - strength training, lifting weights, cardiovascular exercise?)

4. Have you set fitness goals for yourself? If so, what are these goals? 
Where did these goals come from? Can you remember making these goals?

5. What are you hoping to achieve from your participation in fitness?

6. When you are engaging in physical activity (at the gym, at the park, etc.), do you compare your body to other people?

If so, how do you feel? For example, do you feel out of place or uncomfortable around some people? (If so, who?)

7. Are there any specific physical results you would like to see as a result of your participation

in exercise? As you exercise, do you think about "working on" a particular body part(s)?

8. What sources do you rely on, if any, to obtain fitness-related information?

For example, do you read fitness-related magazines, such as Shape, Fitness, or Self? Do you look to fitness books, fitness icons on TV, spiritual guides, etc.? Do you refer to doctors, parents, friends, etc.? Do you believe these sources help you in your fitness goals? If so, how?

Which source do you believe to be the most reliable, and for what reason?

\section{Nutrition-related Questions.}

I would like to ask you questions that are related to your nutritional habits.

1. What is your definition of a healthy diet?

What elements are important to include in one's diet?

2. If a friend asked for advice on his or her diet, would you know what to say? For example, what types of suggestions would you make? Why these particular recommendations?

3. Do you believe your diet is different from or similar to most of your friends' diets? That

is, are you the only vegan or vegetarian that you know? Are you the only one who prefers to eat organic foods? Or rather, do most of your friends eat similarly to you?

4. If you do follow a particular diet (vegan, low-fat, low-carb, etc.), do you recall the primary reason you began eating in this way?

When did you begin following this particular diet?

Is your diet tied to your ideas about health?

5. Is there a certain source upon whom/which you rely for nutrition- or diet-related information (doctors, parent/s, friends, magazines, cookbooks, internet website, fad diet)?

Why this particular source? How did you come to "know" (or find out about) this particular source? 
Upon which source(s) do you rely most heavily for dietary advice? Is there a particular reason for your adherence to this source(s)?

\section{Closing Statements.}

That's the end of the interview. Is there anything else you feel it would be important for us to know to understand your experiences? Do you have any additional thoughts or comments about the issues we have covered? Any questions or comments about the research?

I would like to thank you very much for being so generous with your time and responses. Please feel free to contact me either at my email address - vfrench@gmail.com or at 7447298 - if there is anything you would like to discuss.

Thank you again! 


\section{CURRICULUM VITAE}

Name: Valerie French

Address: Sociology Department

University of Louisville

Louisville, KY 40292

Education: University of Louisville, Louisville KY

Sociology, GPA 3.79

Masters of Arts degree, August 2006

University of Kentucky, Lexington KY

Communication and Information Studies, GPA 3.96

Bachelor of Arts degree, May 2004

Honors: Graduate Assistantship for the Alumni Association at the University of

Louisville-Stipend and $100 \%$ of Tuition

Dean's List, 2000-2001, 2001-2002, 2002-2003, 2003-2004

Recipient of Academic Scholarship, 2002-2003

Recipient of KEES Scholarship, 2000-2001, 2001-2002, 2002-2003, 20032004

Departmental Honors

Golden Key Honor Society Honoree 